\title{
Wavelet filters and infinite-dimensional unitary groups
}

\author{
Ola Bratteli and Palle E. T. Jorgensen
}

\begin{abstract}
In this paper, we study wavelet filters and their dependence on two numbers, the scale $N$ and the genus $g$. We show that the wavelet filters, in the quadrature mirror case, have a harmonic analysis which is based on representations of the $C^{*}$-algebra $\mathcal{O}_{N}$. A main tool in our analysis is the infinite-dimensional group of all maps $\mathbb{T} \rightarrow \mathrm{U}(N)$ (where $\mathrm{U}(N)$ is the group of all unitary $N$-by- $N$ matrices), and we study the extension problem from low-pass filter to multiresolution filter using this group.
\end{abstract}

\section{Contents}

List of Tables

1. Introduction

2. Background on wavelet filters and extensions

3. Decomposition of polynomial loops into linear factors

4. Extensions of low-pass polynomial filters to high-pass filters

5. Representations associated with polynomial filters

6. Reduction of representations as a reduction of $N$

References

\section{LisT OF TABLES}

1 Eigenvalues, multiplicities, and eigenvectors of $\sigma^{(A)}$

21

2 Decompositions from eigenvalue-one multiplicities, and eigenvectors for $\sigma^{(A)}$

\section{Introduction}

Digital filters are defined by a choice of coefficients which weight digital time signals from signal processing. It has been known since the eighties that these coefficients are also those which relate the operations of scaling and translation on the real line $\mathbb{R}$ in the construction of multiresolution wavelets; see (2.11) and (2.18). There the digital filters are also known as quadrature mirror filters and they are

1991 Mathematics Subject Classification. Primary 46L60, 47D25, 42A16, 43A65; Secondary 46L45, 42A65, 41A15.

Key words and phrases. wavelet, Cuntz algebra, representation, orthogonal expansion, quadrature mirror filter, isometry in Hilbert space.

Research supported by the University of Oslo. 
fundamental in generating wavelet bases in $L^{2}(\mathbb{R})$. They can be determined by various rules which however at times appear somewhat ad hoc in the literature. Here we will describe a pairwise bijective correspondence between three sets:

(i) the wavelet filters,

(ii) a certain infinite-dimensional unitary group (also called a loop group), and finally

(iii) a specific class of representations on $L^{2}(\mathbb{T})$ of some relations from $C^{*}$-algebra theory. These last mentioned relations go under the name of the Cuntz relations and are widely studied in operator theory.

The question of reducibility versus irreducibility of the representations in (iii) will be central, as will be unitary equivalence of pairs of representations. Our study of the equivalence question for representations is motivated by a desire for classifying wavelets and systematically constructing interesting examples. The question of reducibility of the representations in turn is motivated by the need for isolating when wavelet data is minimal in a suitable sense. Wavelets come with some specified numerical scaling which may be a natural number, $N=2,3, \ldots$, or it may be an expansive integral matrix of some specified dimension $d$. (Here we shall restrict to $d=1$.) It turns out that the reducibility question for the representations is closely tied to whether or not the scaling data (in this case $N$ ) is minimal in a suitable sense; see Section 6 below.

We also mention that the algebra $\mathcal{O}_{N}$ is used in the theory of superselection sectors in quantum field theory [DoRo89]. While $\mathcal{O}_{N}$ is used there in connection with representation theory, our viewpoint here is completely different: Doplicher and Roberts use in [DoRo87] a category of endomorphisms of $\mathcal{O}_{N}$ in deriving a noncommutative Pontryagin duality theory, also called Tannaka-Krein theory, in giving an algebraic description of $\hat{G}$ when $G$ is a given compact (non-abelian) Lie group. The intertwiners for systems of representations of $G$ in DoRo89 and DoRo87 induce the endomorphisms of $\mathcal{O}_{N}$. Our viewpoint is in a sense the opposite: We identify a class of representations of $\mathcal{O}_{N}$ which has the structure of a compact loop group, i.e., the group of all maps from $\mathbb{T}$ into $\mathrm{U}(N)$ where $\mathrm{U}(N)$ denotes the group of unitary $N \times N$ matrices.

\section{Background on wavelet filters and extensions}

To fix notation, let us give a short rundown of the standard multiresolution wavelet analysis of scale $N$. We follow Section 10 in BrJo97b, but see also GrMa92], [Mey87, [MRV96] and Hör95]. Define scaling by $N$ on $L^{2}(\mathbb{R})$ by

$$
(U \xi)(x)=N^{-\frac{1}{2}} \xi\left(N^{-1} x\right),
$$

and translation by 1 on $L^{2}(\mathbb{R})$ by

$$
(T \xi)(x)=\xi(x-1) \text {. }
$$

A scaling function is a function $\varphi \in L^{2}(\mathbb{R})$, such that if $\mathcal{V}_{0}$ is the closed linear span of all translates $T^{k} \varphi, k \in \mathbb{Z}$, then $\varphi$ has the following four properties:

$$
\begin{aligned}
& \left\{T^{k} \varphi ; k \in \mathbb{Z}\right\} \text { is an orthonormal set in } L^{2}(\mathbb{R}) ; \\
& U \varphi \in \mathcal{V}_{0} ; \\
& \bigwedge_{n \in \mathbb{Z}} U^{n} \mathcal{V}_{0}=\{0\} ;
\end{aligned}
$$




$$
\bigvee_{n \in \mathbb{Z}} U^{n} \mathcal{V}_{0}=L^{2}(\mathbb{R})
$$

The simplest example of a scaling function is the Haar function $\varphi$, which is the characteristic function of the interval $[0,1]$.

By (2.3) we may define an isometry

$$
\mathcal{F}_{\varphi}: \mathcal{V}_{0} \longrightarrow L^{2}(\mathbb{T}): \xi \longrightarrow m
$$

as follows:

$$
\begin{aligned}
& \xi(\cdot)=\sum_{n} b_{n} \varphi(\cdot-n) \\
& \mathcal{F}_{\varphi} \downarrow \\
& m(t)=m\left(e^{-i t}\right)=\sum_{n} b_{n} e^{-i n t} .
\end{aligned}
$$

Then

$$
\hat{\xi}(t)=m(t) \hat{\varphi}(t)
$$

where $\xi \mapsto \hat{\xi}$ is the Fourier transform. If $\xi \in \mathcal{V}_{-1}=U^{-1} \mathcal{V}_{0}$, then $U \xi \in \mathcal{V}_{0}$, so we may define

$$
m_{\xi}=\mathcal{F}_{\varphi}(U \xi) \in L^{2}(\mathbb{T})
$$

and then

$$
\sqrt{N} \hat{\xi}(N t)=m_{\xi}(t) \hat{\varphi}(t)
$$

In particular, defining

$$
m_{0}(t)=m_{\varphi}(t)
$$

we note that condition (2.3) is equivalent to

$$
\operatorname{PER}\left(|\hat{\varphi}|^{2}\right)(t):=\sum_{k}|\hat{\varphi}(t+2 \pi k)|^{2}=(2 \pi)^{-1}, \quad \text { constancy a.e. } t \in \mathbb{R},
$$

which in turn implies

$$
\sum_{k=0}^{N-1}\left|m_{0}(t+2 \pi k / N)\right|^{2}=N .
$$

(Note that (2.14) does not conversely imply (2.13). Condition (2.14) merely implies that $\varphi$ defines a tight frame. In the representation theory in Section 5 this distinction does not play a role. Only the unitarity of $M(z)$ in 2.22 ) is relevant. See Hör95, Theorem 3.3.6]. Similarly, the representation theory in Section 5 applies in cases more general than the ones tied to the $L^{2}(\mathbb{R})$ wavelets via $(2.12)$ and (2.18). In the latter case one also has the low-pass condition $m_{0}(1)=\sqrt{N}$. In the loop representation 2.23)-(2.24), this is equivalent to $A_{0, j}(1)=1 / \sqrt{N}$ for $j=0, \ldots, N-1$. These conditions involve the evaluation of the functions $m_{0}(\cdot)$ or $A_{0, j}(\cdot)$ at $z=1$. But $z=e^{-i \omega}$, where $\omega$ is the frequency variable, so the conditions are the low-pass conditions for $\omega \sim 0$.)

If $\xi, \eta \in U^{-1} \mathcal{V}_{0}$, then we have the equivalence:

$$
\begin{gathered}
\xi \perp T^{k} \eta \text { for all } k \in \mathbb{Z} \\
\mathbb{1} \\
\sum_{k=0}^{N-1} \overline{m_{\xi}(t+2 \pi k / N)} m_{\eta}(t+2 \pi k / N)=0 \text { for almost all } t \in \mathbb{R} .
\end{gathered}
$$


If $\xi \in U^{-1} \mathcal{V}_{0}$, then

$$
\begin{gathered}
\xi(\cdot-k), k \in \mathbb{Z}, \underset{\mathbb{1}}{\text { is an orthonormal set }} \\
\sum_{k=0}^{N-1}\left|m_{\xi}(t+2 \pi k / N)\right|^{2}=N .
\end{gathered}
$$

With the low-pass filter $m_{0}$ already given, now choose high-pass filters $m_{1}, \ldots, m_{N-1}$ in $L^{2}(\mathbb{T})$ such that

$$
\sum_{k=0}^{N-1} \overline{m_{i}(t+2 \pi k / N)} m_{j}(t+2 \pi k / N)=\delta_{i, j} N
$$

for almost all $t \in \mathbb{R}$, and define wavelet functions $\psi_{1}, \ldots, \psi_{N-1}$ by

$$
\sqrt{N} \hat{\psi}_{i}(N t)=m_{i}(t) \hat{\varphi}(t)
$$

Using (2.15)-(2.18) one then shows that the functions

$$
\left\{T^{k} \psi_{i} ; k \in \mathbb{Z}, i=1, \ldots, N-1\right\}
$$

form an orthonormal basis for $\mathcal{V}_{-1} \cap \mathcal{V}_{0}{ }^{\perp}$, and thus, using (2.5)-(2.6), the set

$$
\left\{U^{n} T^{k} \psi_{i} ; n, k \in \mathbb{Z}, i=1, \ldots, N-1\right\}
$$

forms an orthonormal basis for $L^{2}(\mathbb{R})$. Concretely, the functions in 2.20 are

$$
U^{n} T^{k} \psi_{i}(x)=N^{-\frac{n}{2}} \psi_{i}\left(N^{-n} x-k\right) .
$$

Thus, reformulating (2.17), orthonormality of $\left\{U^{n} T^{k} \psi_{i}\right\}$ is equivalent to unitarity of the matrix

$$
M(z)=\frac{1}{\sqrt{N}}\left(\begin{array}{cccc}
m_{0}(z) & m_{0}(\rho z) & \cdots & m_{0}\left(\rho^{N-1} z\right) \\
m_{1}(z) & m_{1}(\rho z) & \cdots & m_{1}\left(\rho^{N-1} z\right) \\
\vdots & & & \\
m_{N-1}(z) & m_{N-1}(\rho z) & \cdots & m_{N-1}\left(\rho^{N-1} z\right)
\end{array}\right)
$$

for almost all $z \in \mathbb{T}$, where $\rho=e^{\frac{2 \pi i}{N}}$ (so it is enough to consider $z=e^{i x}, 0 \leq x<$ $\left.\frac{2 \pi}{N}\right)$. Here $M$ is a function from $\mathbb{T}$ into $\mathrm{U}(N)$ of a special kind. It will be convenient for our analysis to consider general functions from $\mathbb{T}$ into $\mathrm{U}(N)$. To this end, we do a Fourier analysis over the cyclic group $\mathbb{Z} / N \mathbb{Z}$, and we introduce

$$
A_{i, j}(z)=\frac{1}{N} \sum_{w^{N}=z} w^{-j} m_{i}(w)
$$

and the inverse transform

$$
m_{i}(z)=\sum_{j=0}^{N-1} z^{j} A_{i, j}\left(z^{N}\right) .
$$

These transforms are also the key to the correspondences (间) $\Leftrightarrow$ (国) $\Leftrightarrow$ (iii) mentioned in the Introduction. Then (2.24) may be summarized as

$$
M(z)=A\left(z^{N}\right) \frac{1}{\sqrt{N}}\left(\begin{array}{cccc}
1 & 1 & \cdots & 1 \\
z & \rho z & \cdots & \rho^{N-1} z \\
\vdots & \vdots & & \vdots \\
z^{N-1} & \rho^{N-1} z^{N-1} & \cdots & \rho^{(N-1)^{2}} z^{N-1}
\end{array}\right) ;
$$


and the requirement that $M(z)$ is unitary for all $z \in \mathbb{T}$ is now equivalent to $A(z)$ being unitary for all $z \in \mathbb{T}$. But $A$ is an arbitrary loop. (A loop is by definition a function from $\mathbb{T}$ into $\mathrm{U}(N)$. See the introduction to Section 3 .) Hence the formulas (2.23)-(2.24) represent the bijection (1)-(ii) alluded to in the Introduction above.

One main problem considered in this paper is the problem whether the highpass filters $m_{1}, \ldots, m_{N-1}$ selected such that (2.17) is valid can be chosen to be "nice" functions when the low-pass filter $m_{0}$ is "nice", where "nice" may mean continuous, differentiable, polynomial, etc.

Using (2.23), this problem amounts to choosing a unitary matrix $A(z)$ once the first row of $A(z)$ is given as a unit row vector $a(z)$. Ideally, one would like to use a selection map $F$ from the unit sphere $S^{2 N-1}$ of $\mathbb{C}^{N}$ into $N$-dimensional orthogonal frames in $\mathbb{C}^{N}$, i.e.,

$$
\mathbf{F}(\mathbf{x})=\left(\begin{array}{c}
\mathbf{F}_{0}(\mathbf{x}) \\
\vdots \\
\mathbf{F}_{N-1}(\mathbf{x})
\end{array}\right),
$$

where the vectors $\mathbf{F}_{0}(\mathbf{x}), \ldots, \mathbf{F}_{N-1}(\mathbf{x})$ form an orthonormal basis for $\mathbb{C}^{N}$ for each $\mathbf{x} \in S^{2 N-1}$ and such that $\mathbf{F}_{0}(\mathbf{x})=\mathbf{x}$ for each $\mathbf{x}$, and define

$$
A(z)=\left(\begin{array}{c}
a(z) \\
\mathbf{F}_{1}(a(z)) \\
\vdots \\
\mathbf{F}_{N-1}(a(z))
\end{array}\right) .
$$

For example, when $N=2$ one may use Daubechies's choice

$$
\mathbf{F}_{1}\left(x_{1}, x_{2}\right)=\left(-\bar{x}_{2}, \bar{x}_{1}\right) .
$$

In general one can of course find measurable functions with these properties, but it is remarkable that one cannot choose them to be continuous when $N>4$. Following Remark 10.2 in $\left[\mathbf{B r J o 9 7 b}\right.$, if $S^{n-1}$ is the unit sphere in $\mathbb{R}^{n}$, then a theorem of Adams Ada62 says that the highest number of pointwise linearly independent vector fields that can be defined on $S^{n-1}$ is $\rho(n)-1$, where the function $\rho(n)$ is defined as follows: Let $b$ be the multiplicity of 2 in the prime decomposition of $n$; write $b=c+4 d$ where $c \in\{0,1,2,3\}$ and $d \in\{0,1,2, \ldots\}$; and put $\rho(n)=2^{c}+8 d$. Thus one verifies $\rho(2)=2, \rho(4)=4, \rho(8)=8, \rho(16)=9$, and in general $\rho(n)<$ $n$ if $n \notin\{2,4,8\}$. Hence the only possibilities of finding a continuous selection function $F$ are when $N=2$ or $N=4$. When $N=2$ one can use Daubechies's selection function (2.28). When $N=4$ one could possibly use the Cayley numbers to construct a selection function, but we have not been able to verify this. Note that if $\mathrm{U}(N)$ is replaced by the real orthogonal group $\mathrm{O}(N)$, a selection function can be found if and only if $N \in\{2,4,8\}$ by using the complex numbers, the quaternions and the Cayley numbers, respectively. For example, for $N=4$, the matrix

$$
\left(\begin{array}{rrrr}
z_{1} & z_{2} & z_{3} & z_{4} \\
-z_{2} & z_{1} & -z_{4} & z_{3} \\
-z_{3} & z_{4} & z_{1} & -z_{2} \\
-z_{4} & -z_{3} & z_{2} & z_{1}
\end{array}\right)
$$


is in $\mathrm{O}(4)$ whenever the first row is a unit vector in $\mathbb{R}^{4}$. Using the identification of $\mathbb{C}$ as $2 \times 2$ real matrices

$$
z_{1}+i z_{2} \approx\left(\begin{array}{rr}
z_{1} & z_{2} \\
-z_{2} & z_{1}
\end{array}\right)
$$

this corresponds to Daubechies's selection function (2.28). We do not know whether or not there is a selection function in the complex case when $N=4$. The claim made in [BrJo97b, Remark 10.2] that there is such a function for $N=8$ is definitely erroneous. What is true is that the matrix

$$
\left(\begin{array}{rrrrrrrr}
z_{1} & z_{2} & z_{3} & z_{4} & z_{5} & z_{6} & z_{7} & z_{8} \\
-z_{2} & z_{1} & -z_{4} & z_{3} & -z_{6} & z_{5} & z_{8} & -z_{7} \\
-z_{3} & z_{4} & z_{1} & -z_{2} & -z_{7} & -z_{8} & z_{5} & z_{6} \\
-z_{4} & -z_{3} & z_{2} & z_{1} & -z_{8} & z_{7} & -z_{6} & z_{5} \\
-z_{5} & z_{6} & z_{7} & z_{8} & z_{1} & -z_{2} & -z_{3} & -z_{4} \\
-z_{6} & -z_{5} & z_{8} & -z_{7} & z_{2} & z_{1} & z_{4} & -z_{3} \\
-z_{7} & -z_{8} & -z_{5} & z_{6} & z_{3} & -z_{4} & z_{1} & z_{2} \\
-z_{8} & z_{7} & -z_{6} & -z_{5} & z_{4} & z_{3} & -z_{2} & z_{1}
\end{array}\right)
$$

is in $\mathrm{O}(8)$ whenever the first row is a unit vector in $\mathbb{R}^{8}$. However, trying to convert this to an element of $\mathrm{U}(4)$ by the same trick as above leads to

$$
\left(\begin{array}{rrrr}
z_{1} & z_{2} & z_{3} & z_{4} \\
-\overline{z_{2}} & \overline{z_{1}} & -z_{4} & z_{3} \\
-\overline{z_{3}} & z_{4} & \overline{z_{1}} & -z_{2} \\
-z_{4} & -\overline{z_{3}} & \overline{z_{2}} & z_{1}
\end{array}\right),
$$

but this is not in general unitary when the first row is a unit vector in $\mathbb{C}^{4}$.

However, there is a completely different method of selecting $A(z)$ using more global properties of the first row $a(z)$ which applies in the case that $a$ is a polynomial in $z$. This special case is interesting because it corresponds to compactly supported scaling functions $\varphi$ : the scaling function $\varphi$ constructed from $m_{0}$ by cascade approximation has support in $[0, N g-1]$ if and only if the low-pass filter $m_{0}(z)$ is a polynomial of degree at most $g-1$, and satisfies $m_{0}(1)=\sqrt{N}$. See BrJo97b , BrJo98], BrJo99], [BEJ99], ReWe98, and [Dau92]. In this context there is a method of extending $a(z)$ to $A(z)$ which is independent of the selection theory, and $A(z)$ may even be taken to be a polynomial in $z$ (with coefficients in $\mathcal{B}\left(\mathbb{C}^{N}\right)$ ) of degree equal to the degree of $a$. The embryonic form of this method is due to Pollen [Pol90], [Pol92], but has been developed further: see, e.g., ReWe98, Vai93. We will give an account of the method in operator-theoretic form, and give the results in Proposition 3.2 and Theorem 4.1. Combining Theorem 4.1 with (2.25) we therefore obtain

Theorem 2.1. Let $N \in\{2,3, \ldots\}$ and let $\varphi$ be a scaling function with support in $[0, N g-1]$ where $g \in\{1,2,3, \ldots\}$. Then one can use multiresolution wavelet analysis to find associated wavelet functions $\psi_{1}, \ldots, \psi_{N-1}$ also having support in $[0, N g-1]$.

Proof. The conditions on $\varphi, \psi_{1}, \ldots, \psi_{N-1}$ correspond to the functions $m_{0}$, $m_{1}, \ldots, m_{N-1}$ being polynomials of degree $\mathrm{Ng}-1$, Hör95, hence Theorem 2.1 follows from Theorem 4.1 and (2.25). More details are given in Corollary 4.2 and its proof. 
In Sections 5 and 6 we will consider the representations of the Cuntz algebra $\mathcal{O}_{N}$ defined by $m_{0}, m_{1}, \ldots, m_{N-1}$ when these are polynomials, and thus extend results from [BEJ99].

\section{Decomposition of polynomial loops into linear factors}

We define a loop to be a continuous function from the circle $\mathbb{T}$ into the compact group $\mathrm{U}(N)$ of unitary $N \times N$ matrices, where $N=1,2, \ldots$ The set $C(\mathbb{T}, \mathrm{U}(N))$ of loops has a natural group structure under pointwise multiplication, and this group is called the loop group [PrSe86]. We say that a loop is polynomial if its matrix elements are polynomials in the basic variable $z \in \mathbb{T}=\{w \in \mathbb{C} ;|w|=1\}$. The set $\mathcal{P}(\mathbb{T}, \mathrm{U}(N))$ of polynomial loops then forms a semigroup in $C(\mathbb{T}, \mathrm{U}(N))$ called the polynomial loop semigroup. (Note that the formulas (2.23) $-(2.24)$ for the bijection between the set of wavelet filters and the loop group are valid also in the wider category of $L^{\infty}$-functions, i.e., when each $m_{i}^{(A)} \in L^{\infty}(\mathbb{T})$ and each $A_{i, j} \in L^{\infty}(\mathbb{T})$. This is the generality of $[\mathbf{B r J o 9 7 b}$.) Note that if $U$ is a given loop, then $U$ has a class in the $K_{1}$-group of $C\left(\mathbb{T}, M_{N}\right)$, i.e., $K_{1}\left(C\left(\mathbb{T}, M_{N}\right)\right) \cong \mathbb{Z}$, see Bla86. This class is called the McMillan degree in the wavelet literature [ReWe98, and we will denote it by $K_{1}(U)$. It can be computed as the winding number of the map

$$
\mathbb{T} \ni z \longrightarrow \operatorname{det}(U(z)) \in \mathbb{T} .
$$

If $U$ is in the loop semigroup, the integer $K_{1}(U)$ is necessarily nonnegative. This follows from Lemma 3.1, below, applied to $u(z)=\operatorname{det}(U(z))$. The map $U \rightarrow$ $K_{1}(U)$ is a group homomorphism from $C(\mathbb{T}, \mathrm{U}(N))$ onto $\mathbb{Z}$. We need an elementary lemma [ReWe98].

Lemma 3.1. If $u: \mathbb{T} \rightarrow \mathbb{T}$ is a polynomial, then $u$ is a monomial.

Proof. We have $u=\sum_{k=0}^{n} c_{k} z^{k}$ for suitable coefficients $c_{k} \in \mathbb{Z}$. By multiplying $u$ by a nonpositive power of $z$ we may assume $c_{0} \neq 0$. But then $1=u(z) \overline{u(z)}=$ $c_{n} \overline{c_{0}} z^{n}+$ powers of $z$ between $-(n-1)$ and $(n-1)+\overline{c_{n}} c_{0} z^{-n}$ and hence $c_{n}=0$. By induction, $u(z)=c_{0}$.

We now use this to show that any polynomial loop $U$ has a decomposition into linear factors [ReWe98, pp. 60-61].

Proposition 3.2. Let $U$ be a polynomial loop in $\mathcal{P}(\mathbb{T}, \mathrm{U}(N))$. The following conditions are equivalent.

$$
K_{1}(U)=d \text {. }
$$

There exist one-dimensional projections $P_{1}, \ldots, P_{d}$ on $\mathbb{C}^{N}$ and a $V \in \mathrm{U}(N)$ such that

$$
U(z)=U_{1}(z) U_{2}(z) \cdots U_{d}(z) V
$$

where

$$
U_{i}(z)=z P_{i}+\left(1-P_{i}\right)
$$

$$
\text { for } i=1, \ldots, d \text { and } z \in \mathbb{T} \text {. (If } d=0 \text {, then } U(z)=V \text {.) }
$$

Proof. Since $K_{1}\left(U_{i}\right)=1$ and $K_{1}(V)=0$, the implication (3.3) $\Rightarrow$ (3.2) is obvious. We prove the other implication by using induction with respect to $d$. To this end, we will use the following reduction lemma. 
LEMMA 3.3. Assume that

$$
U(z)=A_{0}+A_{1} z+\cdots+A_{k} z^{k}
$$

defines a $U \in \mathcal{P}(\mathbb{T}, \mathrm{U}(N))$, where $A_{k} \neq 0$ and $k \geq 1$. Then $U$ has a unique decomposition

$$
U(z)=((1-Q)+z Q)\left(B_{0}+B_{1} z+\cdots+B_{k-1} z^{k-1}\right),
$$

where $Q$ is the projection onto the range $A_{k} \mathbb{C}^{N}$ of $A_{k}$ (and then $(1-Q)+z Q$ and $B_{0}+B_{1} z+\cdots+B_{k-1} z^{k-1}$ are in $\left.\mathcal{P}(\mathbb{T}, \mathrm{U}(N))\right)$.

Proof. Let $Q$ be the projection onto $A_{k} \mathbb{C}^{N}$. We have

$$
\begin{aligned}
1 & =U(z)^{*} U(z) \\
& =\left(A_{0}^{*}+A_{1}^{*} z^{-1}+\cdots+A_{k}^{*} z^{-k}\right)\left(A_{0}+A_{1} z+\cdots+A_{k} z^{k}\right) \\
& =A_{k}^{*} A_{0} z^{-k}+\text { terms in higher powers of } z .
\end{aligned}
$$

Hence

$$
A_{k}^{*} A_{0}=0,
$$

and thus, since $Q$ is the projection onto the range of $A_{k}$, we obtain

$$
Q A_{0}=0 \quad \text { and } \quad(1-Q) A_{k}=0 .
$$

Now put

$$
\begin{aligned}
W(z)= & ((1-Q)+z Q)^{-1} U(z) \\
= & \left((1-Q)+z^{-1} Q\right)\left(A_{0}+\cdots+z^{k} A_{k}\right) \\
= & z^{-1} Q A_{0} \\
& +\left((1-Q) A_{0}+Q A_{1}\right) \\
& +\cdots \\
& +z^{k}(1-Q) A_{k} \quad \text { (ordered by increasing powers). }
\end{aligned}
$$

It follows from (3.8) that $W(z)$ has the form

$$
W(z)=B_{0}+B_{1} z+\cdots+B_{k-1} z^{k-1},
$$

and (3.5) follows. (In fact, $B_{0}=(1-Q) A_{0}+Q A_{1}=A_{0}+Q A_{1}, \ldots$, and $B_{k-1}=$ $(1-Q) A_{k-1}+A_{k}$.) By our choice of $Q$, the decomposition (3.5) is unique by (3.8).

End of the Proof of Proposition 3.2. We will prove the proposition by induction with respect to the McMillan index $d$. Assume first that $d \geq 1$ and that the proposition holds for all indices $\leq d-1$, and suppose that

$$
U(z)=A_{0}+A_{1} z+\cdots+A_{k} z^{k}
$$

has index $d$. But by Lemma 3.3, $U$ has a decomposition

$$
U(z)=((1-Q)+z Q) W(z),
$$

where $Q$ is a nonzero projection. But then

$$
\begin{aligned}
d & =K_{1}(U)=K_{1}((1-Q)+z Q)+K_{1}(W(z)) \\
& =\operatorname{dim}(Q)+K_{1}(W(z)),
\end{aligned}
$$


so $K_{1}(W(z)) \leq d-1$. We may then apply the induction hypothesis to $W(z)$. Finally, there exist $\operatorname{dim} Q$ one-dimensional projections $P_{1}, \ldots, P_{\operatorname{dim}(Q)}$ such that $Q=P_{1}+P_{2}+\cdots+P_{\operatorname{dim}(Q)}$, and then

$$
((1-Q)+z Q)=\prod_{n=1}^{\operatorname{dim} Q}\left(\left(1-P_{n}\right)+z P_{n}\right) .
$$

It follows that $U$ has the form (3.3).

Finally, if $K_{1}(U)=0$ and $U$ has the form (3.11), there are two possibilities:

1. $k=0$ and $U$ is a constant unitary matrix. Then $U$ already has the form in (3.3) with $d=0$.

2. $k>0$. Then one may apply Lemma 3.3 to write $U(z)=((1-Q)+z Q) W(z)$ with $Q$ a nonzero projection, but as

$$
0=K_{1}(U)=\operatorname{dim} Q+K_{1}(W)
$$

and $K_{1}(W) \geq 0$, this is impossible.

This ends the proof of Proposition 3.2. The last argument in the induction chain is simpler if we use induction with respect to $k$ rather than induction with respect to $d$.

\section{Extensions of low-pass polynomial filters to high-pass filters}

In this section we will prove Theorem 2.1. This theorem follows from Corollary 4.2. below. Theorem 4.1 states that every vector $\alpha$ in $\mathbb{C}^{N g}$ which satisfies a certain orthogonality condition is the first row of coefficients of some element of the loop group.

Theorem 4.1. Let $\alpha=\left(\alpha_{0}, \alpha_{1}, \ldots, \alpha_{g-1}\right)$ be g row vectors in $\mathbb{C}^{N}$. The following conditions are equivalent.

The vectors satisfy the relations

$$
\sum_{i}\left\langle\alpha_{i} \mid \alpha_{i+j}\right\rangle=0 \quad \text { for } j \neq 0
$$

and

$$
\sum_{i}\left\langle\alpha_{i} \mid \alpha_{i}\right\rangle=1
$$

where we use the convention that $\alpha_{i}=0$ if $i<0$ or $i \geq g$.

There exists a polynomial loop

$$
A(z) \in \mathcal{P}(\mathbb{T}, \mathrm{U}(N))
$$

of degree $g-1$ such that the first row of $A(z)$ is

$$
\sum_{i=0}^{g-1} z^{i} \alpha_{i}
$$

Proof. The implication $(4.2) \Rightarrow(4.1)$ follows from considering the $(0,0)$ matrix entry of

$$
A(z) A(z)^{*}=1
$$

in $M_{N}$ for all $z$, i.e., $A(z) A(z)^{*}$ is the constant Laurent polynomial 1. 
The other implication is proved by a very similar method as in the proof of Proposition 3.2. Again we use induction with respect to $g$.

If $g=1$, condition (4.1) just says that $\alpha_{0}$ is a unit row vector, and we can find a constant function $A(z)=A$ just by Gram-Schmidt orthogonalization.

Assume that $g>1$, and that the result has been proved for all smaller $g$. We may assume that $\alpha_{g-1} \neq 0$ (otherwise we are already through by the induction hypothesis). Let $P$ be the one-dimensional projection onto $\alpha_{g-1}$, i.e.,

$$
\alpha_{g-1} P=\alpha_{g-1}
$$

Now define

$$
\begin{aligned}
\beta(z)=\alpha & (z)\left(1-P+z^{-1} P\right) \\
= & \left(\alpha_{0}+\alpha_{1} z+\cdots+\alpha_{g-1} z^{g-1}\right)\left(1-P+z^{-1} P\right) \\
= & z^{-1} \alpha_{0} P \\
& +\alpha_{0}(1-P)+\alpha_{1} P \\
& +z(\cdots) \\
& +\ldots \\
& +z^{g-2}\left(\alpha_{g-1} P+\alpha_{g-2}(1-P)\right) \\
& +z^{g-1} \alpha_{g-1}(1-P) .
\end{aligned}
$$

But since $\left\langle\alpha_{0} \mid \alpha_{g-1}\right\rangle=0$ by (4.1), the $z^{-1}$ term disappears, and 4.4) implies that the $z^{g-1}$ term disappears. Therefore $\beta(z)$ is a polynomial in $z$ of degree $g-2$. One now verifies from the unitarity of $\left(1-P+z^{-1} P\right)$ that the coefficient vectors of $\beta$ satisfy the same relations (4.1) as those of $\alpha$. Hence, applying the induction hypothesis, there exists a polynomial loop $B(z)$ of degree $g-2$ such that the first row of $B(z)$ is $\beta(z)$. Thus, putting

$$
A(z)=B(z)(1-P+z P),
$$

it follows from (4.5) that the first row of $A(z)$ is $\alpha(z)$. This completes the induction, and thus the proof of Theorem 4.1.

Let us restate Theorem 2.1 and its proof in terms of the low-pass filter $m_{0}(z)$ :

Corollary 4.2. Let $m_{0}$ be a polynomial, and let $N \in\{2,3, \ldots\}$. Suppose

$$
\left|m_{0}(z)\right|^{2}+\left|m_{0}(z \rho)\right|^{2}+\cdots+\left|m_{0}\left(z \rho^{N-1}\right)\right|^{2}=N
$$

for all $z \in \mathbb{T}$, where $\rho=e^{\frac{2 \pi i}{N}}$. Then there are polynomials $\left\{m_{i} ; i=1, \ldots, N-1\right\}$ such that the combined system $\left\{m_{i} ; 0 \leq i<N\right\}$ satisfies the unitarity property of (2.22), or equivalently (2.17) with the convention $z \leftrightarrow e^{i t}$. In other words, every $m_{0}$ may be completed to a quadrature mirror filter system.

Furthermore, when $g \in \mathbb{N}$ is chosen such that the degree of the polynomial $m_{0}$ is at most $N g-1$, then the polynomials $m_{1}, \ldots, m_{N-1}$ can be taken to have degree at most $\mathrm{Ng}-1$.

Proof. With $m_{0}$ and $N$ given as stated in the corollary, set

$$
A_{0, j}(z):=\frac{1}{N} \sum_{w^{N}=z} w^{-j} m_{0}(w), \quad z \in \mathbb{T} .
$$


Then it follows from (4.7) that the coefficients $\alpha_{j}$, as a set of row vectors in $\mathbb{C}^{N}$, satisfy (4.1) in Theorem 4.1. Picking then $A(z)=\left(A_{i, j}(z)\right) \in \mathcal{P}(\mathbb{T}, \mathrm{U}(N))$ as in (4.2), using the theorem, and setting

$$
m_{i}(z)=\sum_{j=0}^{N-1} z^{j} A_{i, j}\left(z^{N}\right),
$$

see (2.24), we note that these functions satisfy the conclusion in the corollary.

Finally, we note that, as in Remark 5.6, if $m_{0}$ has degree at most $N g-1$, it follows from (4.8) that $A_{0, j}(z)$ has degree at most $g-1$; thus $A_{i, j}(z)$ can be taken to have degree at most $g-1$ by Theorem 4.1, and then all $m_{i}(z)$ have degree at most $N g-1$ by Remark 5.6 or (4.9).

\section{Representations associated with polynomial filters}

If $m_{0}, m_{1}, \ldots, m_{N-1}$ are complex functions on $\mathbb{T}$ such that the matrix $M(z)$ in (2.22) is unitary, one may define isometries $S_{0}, \ldots, S_{N-1}$ on $L^{2}(\mathbb{T})$ by

$$
\left(S_{i} \xi\right)(z)=m_{i}(z) \xi\left(z^{N}\right)
$$

and then

$$
\left(S_{i}^{*} \xi\right)(z)=\frac{1}{N} \sum_{\substack{w \\ w^{N}=z}} \bar{m}_{i}(w) \xi(w),
$$

see [BrJo97b, (1.16)-(1.17)]. The isometries $S_{0}, \ldots, S_{N-1}$ then have mutually orthogonal ranges,

$$
S_{i}^{*} S_{j}=\delta_{i, j} \mathbb{1}
$$

and these ranges add up to $\mathbb{1}$,

$$
\sum_{i=0}^{N-1} S_{i} S_{i}^{*}=\mathbb{1} .
$$

The relations (5.3)-(5.4) are called the Cuntz relations (of order $N$ ), and the universal $C^{*}$-algebra generated by operators $s_{0}, \ldots, s_{N-1}$ satisfying these relations is called the Cuntz algebra (of order $N$ ). It is a simple antiliminal $C^{*}$-algebra, which means that its space of Hilbert-space realizations cannot be equipped with a standard Borel structure (see Cun77]).

Let us recall some results from [BJKW97] and [BEJ99]. Let $\mathcal{H}$ be a Hilbert space, and $s_{i} \rightarrow \pi\left(s_{i}\right)=S_{i} \in \mathcal{B}(\mathcal{H})$ a representation of the Cuntz relations on $\mathcal{H}$. Assume that there exists a finite-dimensional subspace $\mathcal{K} \subset \mathcal{H}$ with the properties

$$
S_{i}^{*} \mathcal{K} \subset \mathcal{K}, \quad i=0,1, \ldots, N-1,
$$

and

$$
\mathcal{K} \text { is cyclic, i.e., }\left[\pi\left(\mathcal{O}_{N}\right) \mathcal{K}\right]=\mathcal{H}
$$

(here $\left[\pi\left(\mathcal{O}_{N}\right) \mathcal{K}\right]$ denotes the closure of the linear span of all polynomials in $S_{0}, \ldots, S_{N-1}$ applied to vectors in $\left.\mathcal{K}\right)$. Define operators $V_{i} \in \mathcal{B}(\mathcal{K})$ by

$$
V_{i}^{*}=\left.S_{i}^{*}\right|_{\mathcal{K}}
$$


Then

$$
\sum_{i=0}^{N-1} V_{i} V_{i}^{*}=\mathbb{1}_{\mathcal{K}}
$$

Define a map $\sigma$ on $\mathcal{B}(\mathcal{K})$ by

$$
\sigma(A)=\sum_{i=0}^{N-1} V_{i} A V_{i}^{*} .
$$

Then $\sigma$ is a unital completely positive map, and

THEOREM 5.1. ([BJKW97]) There is a positive norm-preserving linear isomorphism between the commutant algebra

$$
\pi\left(\mathcal{O}_{N}\right)^{\prime}=\left\{A \in \mathcal{B}(\mathcal{H}) ; A \pi(x)=\pi(x) A \text { for all } x \in \mathcal{O}_{N}\right\}
$$

and the fixed-point set

$$
\mathcal{B}(\mathcal{K})^{\sigma}=\{A \in \mathcal{B}(\mathcal{K}) ; \sigma(A)=A\}
$$

given by

$$
\pi\left(\mathcal{O}_{N}\right)^{\prime} \ni A \longrightarrow P A P,
$$

where $P$ is the projection of $\mathcal{H}$ onto $\mathcal{K}$. In particular, $\pi$ is irreducible if and only if $\sigma$ is ergodic.

More generally, if $\mathcal{K}_{1}, \mathcal{K}_{2}$ (with corresponding projections $P^{(1)}$ and $P^{(2)}$ ) are $S^{*}$-invariant cyclic subspaces for two representations $\pi_{1}, \pi_{2}$ of $\mathcal{O}_{N}$ on $\mathcal{H}_{1}, \mathcal{H}_{2}$, and

$$
V_{i}^{(j)}=\left.P^{(j)} \pi_{j}\left(s_{i}\right)\right|_{\mathcal{K}_{j}}
$$

for $j=1,2, i=0, \ldots, N-1$, define $\rho$ on $\mathcal{B}\left(\mathcal{K}_{1}, \mathcal{K}_{2}\right)$ by

$$
\rho(A)=\sum_{i} V_{i}^{(2)} A V_{i}^{(1) *}
$$

Then there is an isometric linear isomorphism between the set of intertwiners

$$
\left\{A \in \mathcal{B}\left(\mathcal{H}_{1}, \mathcal{H}_{2}\right) ; A \pi_{1}(x)=\pi_{2}(x) \text { A for all } x \in \mathcal{O}_{N}\right\}
$$

and the fixed-point set

$$
\left\{B \in \mathcal{B}\left(\mathcal{K}_{1}, \mathcal{K}_{2}\right) ; \rho(B)=B\right\}
$$

given by

$$
A \longrightarrow B=P^{(2)} A P^{(1)} .
$$

We argued in BEJ99, (4.14)-(4.18)] that if $m_{0}, \ldots, m_{N-1}$ are polynomials in the circle variable $z, N=2$, and $\mathcal{H}=L^{2}(\mathbb{T})$, then such a finite-dimensional subspace $\mathcal{K}$ exists, having dimension $N g$ and spanned by $1, z^{-1}, z^{-2}, \ldots, z^{-N g+1}$. In this section we will extend this result to $N>2$, and we will see in Proposition 5.5 that we can do slightly better than what the $N=2$ result indicates. Let us assume from now on that the loop group element $A(z)$ in $(2.23)-(2.25)$ is a polynomial of degree $g-1$, so that $m_{0}(z), \ldots, m_{N-1}(z)$ are polynomials of degree at most $N(g-1)+N-1=N g-1$. 
We will use the notation $A(z)=\left(A_{i, j}(z)\right)_{i, j=0}^{N-1}$ for the loop-group element $A: \mathbb{T} \rightarrow \mathrm{U}(N)$. Since the Fourier expansion is finite, $A(z)$ has the form

$$
A(z)=\sum_{k=0}^{g-1} z^{k} A^{(k)}
$$

where $A^{(k)} \in \mathcal{B}\left(\mathbb{C}^{N}\right)$ for $k=0, \ldots, g-1$. The factorization in Lemma 3.3 motivates the name genus for $g$.

We have

Corollary 5.2. If $A(z)$ is a general polynomial of $z$ with values in $\mathcal{B}\left(\mathbb{C}^{N}\right)$ of the form (5.18), the following four conditions (5.19)-(5.22) are equivalent:

$$
\begin{aligned}
& A(z)^{*} A(z)=\mathbb{1}_{N}, z \in \mathbb{T}, \text { i.e., } A \text { takes values in } \mathrm{U}(N) ; \\
& \sum_{k} A^{(k) *} A^{(k+n)}=\left\{\begin{array}{ll}
\mathbb{1}_{N} & \text { if } n=0, \\
0 & \text { if } n \in \mathbb{Z} \backslash\{0\},
\end{array},\right. \text { with the convention that } \\
& A^{(m)}=0 \text { if } m \notin\{0,1, \ldots, g-1\} ; \\
& \text { there are projections } P_{1}, \ldots, P_{s} \text { in } \mathcal{B}\left(\mathbb{C}^{N}\right), \text { positive integers } \\
& r_{1}, \ldots, r_{s}, \text { and a unitary } W \in \mathrm{U}(N) \text { such that } \\
& A(z)=\left(\prod_{j=1}^{s}\left(\mathbb{1}_{N}-P_{j}+z^{r_{j}} P_{j}\right)\right) W ;
\end{aligned}
$$

and

$$
\begin{aligned}
& \text { there are projections } Q_{0}, Q_{1}, \ldots, Q_{g-1} \text { and a unitary } V \in \mathrm{U}(N) \\
& \text { such that } \\
& A^{(0)}=V \prod_{j=0}^{g-1}\left(\mathbb{1}_{N}-Q_{j}\right), \\
& A^{(1)}=V \sum_{j=0}^{g-1}\left(\mathbb{1}_{N}-Q_{0}\right) \cdots \\
& \cdots\left(\mathbb{1}_{N}-Q_{j-1}\right) Q_{j}\left(\mathbb{1}_{N}-Q_{j+1}\right) \cdots \\
& \cdots\left(\mathbb{1}_{N}-Q_{g-1}\right) \text {, } \\
& A^{(g-1)}=V \prod_{j=0}^{g-1} Q_{j} .
\end{aligned}
$$

Proof. This follows from Proposition 3.2 and explicit computations.

REMARK 5.3. The case $g=2=N$ includes the family of wavelets introduced by Daubechies Dau92 and studied further in [BEJ99. Note that $g=2$ yields the representation

$$
A^{(0)}=V\left(\mathbb{1}_{N}-Q\right), \quad A^{(1)}=V Q,
$$

by $(5.22)$. But then (5.20) takes the form

$$
A^{(0) *} A^{(0)}=\mathbb{1}_{N}-Q, \quad A^{(1) *} A^{(1)}=Q,
$$

which will be used in the sequel. 
Let us return to the connection between loop-group elements and representations of $\mathcal{O}_{N}$. The algebra $\mathcal{O}_{N}$ has the following basic representation $s_{i} \mapsto S_{i}$ on $L^{2}(\mathbb{T})$ :

$$
S_{j} \xi(z)=z^{j} \xi\left(z^{N}\right), \quad 0 \leq j<N, \xi \in L^{2}(\mathbb{T}), z \in \mathbb{T} .
$$

If $s_{i} \mapsto T_{i}$ is any other representation, then as noted in Cun77, Cun80, and BJP96, the system $\left(S_{j}^{*} T_{i}\right)_{i, j}$ realizes a unitary $N \times N$ matrix with entries in $\mathcal{B}\left(L^{2}(\mathbb{T})\right)$.

Proposition 5.4. Let $\left(S_{i}\right)$ be the basic representation of $\mathcal{O}_{N}$, and let $\left(T_{i}\right)$ be an arbitrary representation. Then the following two conditions are equivalent:

$$
\text { Each operator } S_{j}^{*} T_{i} \text { on } L^{2}(\mathbb{T}) \text { is a multiplication operator; }
$$

and

$$
\begin{aligned}
& \text { the } T_{i} \text {-representation has the form } \\
& \qquad T_{i} \xi(z)=m_{i}(z) \xi\left(z^{N}\right), \quad 0 \leq i<N, \xi \in L^{2}(\mathbb{T}), z \in \mathbb{T}, \\
& \text { where } m_{0}, \ldots, m_{N-1} \in L^{\infty}(\mathbb{T}) \text {. }
\end{aligned}
$$

Proof. $(5.26) \Rightarrow(5.27)$ : If 5.26$)$ holds, then there are functions $A_{i, j} \in L^{\infty}(\mathbb{T})$ such that $S_{j}^{*} T_{i}=A_{i, j}$ where the right-hand side also denotes the multiplication operator determined by the function in question. Then

$$
T_{i}=\sum_{j=0}^{N-1} S_{j} S_{j}^{*} T_{i}=\sum_{j=0}^{N-1} S_{j} A_{i, j}=\sum_{j=0}^{N-1} A_{i, j}\left(z^{N}\right) S_{j} .
$$

Setting

$$
m_{i}(z)=\sum_{j=0}^{N-1} A_{i, j}\left(z^{N}\right) z^{j}
$$

and using the Cuntz relations, we conclude that $T_{i}$ has the form in (5.27).

$(5.27) \Rightarrow(5.26)$ : If, conversely, the representation $\left(T_{i}\right)$ is given to have the form in (5.27), then one checks that the filter functions $m_{i}$ must satisfy the unitarity property (2.22) above. If then $A_{i, j}(z)$ are given by (2.23), then

$$
S_{j}^{*} T_{i} \xi(z)=\frac{1}{N} \sum_{w^{N}=z} w^{-j} T_{i} \xi(w)=\frac{1}{N} \sum_{w^{N}=z} w^{-j} m_{i}(w) \xi(z),
$$

and we conclude that (5.26) holds, i.e., $S_{j}^{*} T_{i}$ is multiplication by the function $A_{i, j}(\cdot)$, where $A_{i, j}$ is derived from $m_{i}$ via (2.23)

We will henceforth denote the representation $T_{i}$ defined by $\left(5.30\right.$ by $T_{i}^{(A)}$, so in particular,

$$
T_{i}^{(\mathbb{1})}=S_{i}
$$

where the notation $S_{i}$ is reserved for the representation defined by (5.25).

We will now apply the results in (5.5)-5.17) to analyze these representations further. As mentioned after (5.17), the linear span $\mathcal{K}$ of $1, z^{-1}, z^{-2}, \ldots, z^{-r}$ is cyclic and $T^{*}$-invariant for a suitable $r \in \mathbb{N}$, i.e., satisfies (5.5)-(5.6) with $T$ in lieu of $S$, see [BEJ99, Proposition 3.1 and Corollary 3.3]. If $m_{0}, \ldots, m_{N-1}$ can be derived from a polynomial loop $A(z)$ of degree $g-1$ by (2.24), we may explicitly estimate 
$r$. To this end, let us look at the action of $T_{i}^{(A) *}$ on $e_{n}(z)=z^{n}$. Put $n=j+N l$ uniquely, where $j \in\{0,1, \ldots, N-1\}$ and $l \in \mathbb{Z}$. Then, using (5.28),

$$
\begin{aligned}
T_{i}^{(A) *} e_{n} & =\sum_{j^{\prime}} S_{j^{\prime}}^{*} \overline{A_{i, j^{\prime}}\left(z^{N}\right)} e_{n} \\
& =\sum_{k} \sum_{j^{\prime}} S_{j^{\prime}}^{*} \overline{A_{i, j^{\prime}}^{(k)}} z^{-N k} e_{n}=\sum_{k} \sum_{j^{\prime}} \overline{A_{i, j^{\prime}}^{(k)}} S_{j^{\prime}}^{*} e_{n-N k} .
\end{aligned}
$$

But $n-N k=j+N l-N k=j+N(l-k)$, and since

$$
S_{j^{\prime}}^{*} e_{j+N(l-k)}= \begin{cases}e_{l-k} & \text { if } j^{\prime}=j, \\ 0 & \text { if } j^{\prime} \neq j,\end{cases}
$$

we obtain

$$
T_{i}^{(A) *} e_{j+N l}=\sum_{k=0}^{g-1} \overline{A_{i, j}^{(k)}} e_{l-k} .
$$

It follows that $T_{i}^{(A) *}$ is represented by a matrix which is a slanted block matrix of the following form:

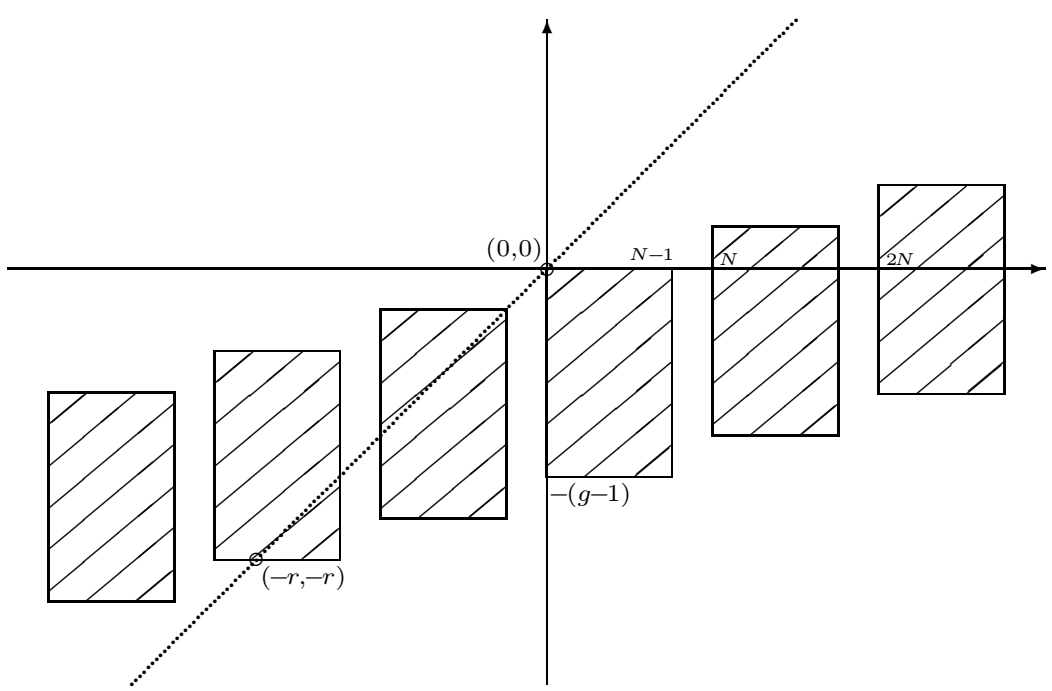

The matrix elements outside the shaded blocks are all zero, and each block has $N$ columns and $g$ rows. Each block is a translate $N$ steps to the right and 1 step up compared to the previous one, and the most central block is located with corners at $(0,0),(N-1,0),(N-1,-g+1),(0,-g+1)$. It follows that if we fix one $n \in \mathbb{Z}$, iterated applications of $T_{i}^{(A) *}$ for various $i$ 's ultimately will transform this vector into a linear combination of $e_{m}$ 's where $m \in\{0,-1,-2, \ldots,-r\}$, where $r$ is the largest integer such that $(-r,-r)$ is contained in one of the blocks. Thus the linear span $\mathcal{K}$ of $\left\{e_{0}, e_{-1}, \ldots, e_{-r}\right\}$ is $T_{i}^{(A) *}$-invariant, and because of the relation

$$
\mathbb{1}=\sum_{\substack{I \\|I|=n}} T_{I}^{(A)} T_{I}^{(A) *},
$$

$\mathcal{K}$ will also be cyclic (see BEJ99, Section 3] for more details of this argument). Thus, computing $r$ more explicitly, we deduce 
Proposition 5.5. If $T^{(A)}$ is the representation of the Cuntz algebra $\mathcal{O}_{N}$ defined by a polynomial loop $A(z)$ of degree $g-1$, then the subspace

$$
\mathcal{K}=\operatorname{lin} \operatorname{span}\left\{e_{0}, e_{-1}, e_{-2}, \ldots, e_{-r}\right\}
$$

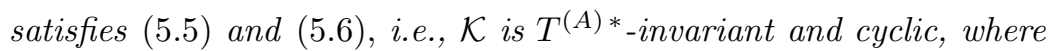

$$
r=g+\left\lfloor\frac{g-1}{N-1}\right\rfloor=\left\lfloor\frac{g N-1}{N-1}\right\rfloor .
$$

Here $\lfloor x\rfloor$ is the largest integer $\leq x$.

Proof. From the figure (5.35) it follows that

$$
r=\left\{\begin{array}{lll}
g & \text { if } g<N, & \text { i.e., } g-1<N-1 \\
g+1 & \text { if } N+1 \leq g+1<2 N, & \text { i.e., } N-1 \leq g-1<2 N-2 \\
g+2 & \text { if } 2 N+1 \leq g+2<3 N, & \text { i.e., } 2 N-2 \leq g-1<3 N-3 \\
\text { etc. } &
\end{array}\right.
$$

Here the first column of ranges ("if ...") is taken from the box diagram (5.35), and the second column of ranges ("i.e., ...") is derived from the first by subtraction of suitable integers. The second column shows how the ratio $\frac{g-1}{N-1}$ arises in (5.38).

REMARK 5.6. Proposition 5.5 could alternatively have been proved by exactly the method used to show (4.18) in BEJ99 from Remark 3.2 there, using joint invariant sets for the maps $n \rightarrow(n-k) / N$ for $k=0,1, \ldots, g N-1$. Note that a consequence of (2.23) and (2.24) is that the degree of all the polynomials $A_{i, j}(z)$ for $i, j=0, \ldots, N-1$ is at most $g-1$ if and only if the degree of all the polynomials $m_{i}(z)$ for $i=0, \ldots, N-1$ is at most $g N-1$.

The case considered in detail in BEJ99 was $N=g=2$, so we regain the result $r=3$ from there, i.e., in that case $\mathcal{K}=\operatorname{span}\left\{e_{0}, e_{-1}, e_{-2}, e_{-3}\right\}$. Note that the denominator $N-1$ in $(5.38)$ shows the dependence of $r=(\operatorname{dim} \mathcal{K})-1$ on the scaling number $N$ for the given wavelet filter. So when $N>2$, the denominator $N-1$ in (5.38) yields a smaller value for $r$ than the value $g N-1$ from [BEJ99] in the special case $N=2$.

We will now apply Theorem 5.1 to the representations above, when $\mathcal{K}=\mathcal{K}^{(A)}$ is given by (5.37) and $r=r^{(A)}$ by (5.38). The operators $V_{i} \in \mathcal{B}\left(\mathcal{K}^{(A)}\right)$ defined by $T_{i}^{(A)}$ in lieu of $S_{i}$ in 5.7 will be denoted by $V_{i}^{(A)}$, and then the $\sigma$ defined by 5.9 is denoted by $\sigma^{(A)}=\sigma^{(A, A)}$, i.e.,

$$
\sigma^{(A, A)}(X)=\sum_{i=0}^{N-1} V_{i}^{(A)} X V_{i}^{(A) *}
$$

for $X \in \mathcal{B}\left(\mathcal{K}^{(A)}\right)$. If $T^{(A)}, T^{(B)}$ are two representations of our kind, let $\sigma^{(B, A)}$ denote the $\rho$ defined by 5.14$)$, i.e.,

$$
\sigma^{(B, A)}(X)=\sum_{i} V_{i}^{(B)} X V_{i}^{(A) *}
$$

so $\sigma^{(B, A)}$ is in $\mathcal{B}\left(\mathcal{K}^{(A)}, \mathcal{K}^{(B)}\right)$. We will mostly be interested in the situation that $A$ and $B$ have the same genus $g$, and then $\mathcal{K}^{(A)}=\mathcal{K}^{(B)}$ by Proposition 5.5 (of course we may always replace $g^{(A)}, g^{(B)}$ by $\max \left\{g^{(A)}, g^{(B)}\right\}$ and thus assume $\mathcal{K}^{(A)}=$ 
$\left.\mathcal{K}^{(B)}\right)$. Now $\mathcal{B}\left(\mathcal{K}^{(A)}, \mathcal{K}^{(B)}\right)$ can be made into a Hilbert space $\mathcal{K}^{(B, A)}$ in a natural fashion by defining the norm of $\psi \in \mathcal{B}\left(\mathcal{K}^{(A)}, \mathcal{K}^{(B)}\right)$ by

$$
\|\psi\|_{2}^{(B, A)}=\operatorname{Tr}^{(A)}\left(\psi^{*} \psi\right)=\operatorname{Tr}^{(B)}\left(\psi \psi^{*}\right),
$$

where $\operatorname{Tr}^{(A)}, \operatorname{Tr}^{(B)}$ are the standard unnormalized traces on $\mathcal{B}\left(\mathcal{K}^{(A)}\right), \mathcal{B}\left(\mathcal{K}^{(B)}\right)$, respectively. Then $\sigma^{(B, A)} \in \mathcal{B}\left(\mathcal{K}^{(B, A)}\right)$. Because of Theorem 5.1, our main concern will be the spectral multiplicity of 1 in the spectrum $\operatorname{Sp}\left(\sigma^{(B, A)}\right)$, as well as the associated eigensubspace $\mathcal{K}^{(B, A)}$. It turns out that these items are much simpler to compute for the adjoint $\sigma^{(B, A) *} \in \mathcal{B}\left(\mathcal{K}^{(B, A)}\right)$ (i.e., the adjoint of $\sigma^{(B, A)}$ as a Hilbert-space operator). Then $\operatorname{Sp}\left(\sigma^{(B, A) *}\right)=\overline{\mathrm{Sp}\left(\sigma^{(B, A)}\right)}$, and in particular $1 \in \operatorname{Sp}\left(\sigma^{(B, A)}\right)$ if and only if $1 \in \operatorname{Sp}\left(\sigma^{(B, A) *}\right)$. If $A=B$ and the completely positive unital map $\sigma^{(A, A)}$ admits a faithful invariant state, it follows from BrJo97a, Lemma 6.3] that the dimensions of the fixed-point sets of $\sigma^{(A, A)}$ and $\sigma^{(A, A) *}$ are the same. However, we will see that $\sigma^{(A, A)}$ does not necessarily admit a faithful invariant state. Our strategy will be to compute $\operatorname{Sp}\left(\sigma^{(B, A)}\right)$ by evaluating

$$
\operatorname{det}\left(x \mathbb{1}-\sigma^{(A, B) *}\right)=\overline{\operatorname{det}\left(\bar{x} \mathbb{1}-\sigma^{(A, B)}\right)}
$$

and then compute the fixed points by hand. Note that from (5.41) we have

$$
\sigma^{(B, A) *}(X)=\sum_{i} V_{i}^{(B) *} X V_{i}^{(A)}
$$

for $X \in \mathcal{K}^{(B, A)}=\mathcal{B}\left(\mathcal{K}^{(A)}, \mathcal{K}^{(B)}\right)$. If $X=E_{-k,-l}=$ the rank-one partial isometry mapping $e_{-l}$ into $e_{-k}$, we compute, in Dirac's notation,

$$
\sigma^{(B, A)}\left(E_{-k,-l}\right)=\sum_{i} V_{i}^{(B)} E_{-k,-l} V_{i}^{(A) *}=\sum_{i}\left|V_{i}^{(B)} e_{-k}\right\rangle\left\langle V_{i}^{(A)} e_{-l}\right|,
$$

which is complicated to handle. On the other hand,

$$
\begin{aligned}
& \sigma^{(B, A) *}\left(E_{-k,-l}\right)=\sum_{i} V_{i}^{(B) *} E_{-k,-l} V_{i}^{(A)} \\
& =\sum_{i}\left|V_{i}^{(B) *} e_{-k}\right\rangle\left\langle V_{i}^{(A) *} e_{-l}\left|=\sum_{i}\right| T_{i}^{(B) *} e_{-k}\right\rangle\left\langle T_{i}^{(A) *} e_{-l}\right|,
\end{aligned}
$$

where the last step used $T^{*}$-invariance of $\mathcal{K}$, see (5.5) and (5.7). But $T^{*}$ is given by (5.34), and hence the matrix elements of $\sigma^{(B, A)}$ can be computed explicitly. Let us do the calculation in a special case:

The case $g=2$ and $N>g$. Then $r=2$ by Proposition 5.5, and $\mathcal{K}$ is three-dimensional. Then the loop $A(z)$ has the form

$$
A(z)=V(1-Q+z Q)=A^{(0)}+z A^{(1)},
$$

where $V$ is a unitary and $Q$ a projection in $\mathcal{B}\left(\mathbb{C}^{N}\right)$. As noted in (5.24), we have

$$
A^{(0) *} A^{(0)}=\mathbb{1}_{N}-Q, \quad A^{(1) *} A^{(1)}=Q .
$$

Now define

$$
\lambda_{i, j}=(\mathbb{1}-Q)_{i, j}=\delta_{i, j}-Q_{i, j}, \quad i, j=0,1,2, \ldots, N-1 .
$$


Setting $\lambda_{i}:=\lambda_{i, i}$, then (5.46) and (5.34) give

$$
\left\{\begin{array}{l}
\sigma^{(A, A) *}\left(E_{0,0}\right)=\lambda_{0} E_{0,0}+\left(1-\lambda_{0}\right) E_{-1,-1}, \\
\sigma^{(A, A) *}\left(E_{-1,-1}\right)=\lambda_{N-1} E_{-1,-1}+\left(1-\lambda_{N-1}\right) E_{-2,-2}, \\
\sigma^{(A, A) *}\left(E_{-2,-2}\right)=\lambda_{N-2} E_{-1,-1}+\left(1-\lambda_{N-2}\right) E_{-2,-2}, \\
\sigma^{(A, A) *}\left(E_{0,-1}\right)=\lambda_{0, N-1} E_{0,-1}-\lambda_{0, N-1} E_{-1,-2}, \\
\sigma^{(A, A) *}\left(E_{0,-2}\right)=\lambda_{0, N-2} E_{0,-1}-\lambda_{0, N-2} E_{-1,-2}, \\
\sigma^{(A, A) *}\left(E_{-1,-2}\right)=\lambda_{N-1, N-2} E_{-1,-1}-\lambda_{N-1, N-2} E_{-2,-2}, \\
\sigma^{(A, A) *}\left(E_{-k,-l}\right)=\sigma^{(A, A) *}\left(E_{-l,-k}\right) \quad \text { whenever } 0 \leq l<k \leq 2 .
\end{array}\right.
$$

From here one computes

$$
\begin{aligned}
& \operatorname{det}\left(x \mathbb{1}-\sigma^{(A, A)}\right)=\operatorname{det}\left(x \mathbb{1}-\sigma^{(A, A) *}\right) \\
& =x^{4}(x-1)\left(x-\lambda_{0}\right)\left(x-\left(\lambda_{N-1}-\lambda_{N-2}\right)\right)\left(x-\lambda_{0, N-1}\right)\left(x-\overline{\lambda_{0, N-1}}\right) .
\end{aligned}
$$

Since the $\lambda_{i, j}$ are matrix elements of a projection, we see immediately that the eigenvalue 1 has multiplicity one except in special cases as when $\lambda_{0}=1, \lambda_{N-1}=1$ and $\lambda_{N-2}=0$, or $\lambda_{0, N-1}=1$. Hence:

THEOREM 5.7. If $g=2$ and $N>2$, the representation $T^{(A)}$ is irreducible for generic loops $A$. It is reducible if and only if the projection $Q \in \mathcal{B}\left(\mathbb{C}^{N}\right)$ defining $A$ by (5.47) satisfies at least one of the conditions

$$
Q_{0,0}=0 \quad\left(\Longleftrightarrow \lambda_{0}=1\right)
$$

or

$$
Q_{N-1, N-1}=0 \quad \text { and } \quad Q_{N-2, N-2}=1 \quad\left(\Longleftrightarrow \lambda_{N-1}=1 \text { and } \lambda_{N-2}=0\right) \text {. }
$$

Proof. We will use the fact that the $\lambda_{i, j}$ numbers are the matrix entries of a projection, $\mathbb{1}-Q$. Since $(\mathbb{1}-Q)^{2}=(\mathbb{1}-Q)$, we have

$$
\lambda_{k}=\lambda_{k}^{2}+\sum_{j \neq k}\left|\lambda_{k, j}\right|^{2}
$$

Thus the case 5.52 may occur with

$$
Q=\left(\begin{array}{c|cccc}
0 & 0 & 0 & \cdots & 0 \\
\hline 0 & & \vdots & \cdots & \vdots \\
0 & & \vdots & & \\
\vdots & & Q_{\mathrm{red}} & \vdots \\
0 & \cdots & \cdots & \cdots
\end{array}\right)=(0) \oplus Q_{\mathrm{red}}
$$

Also (5.53) may occur with

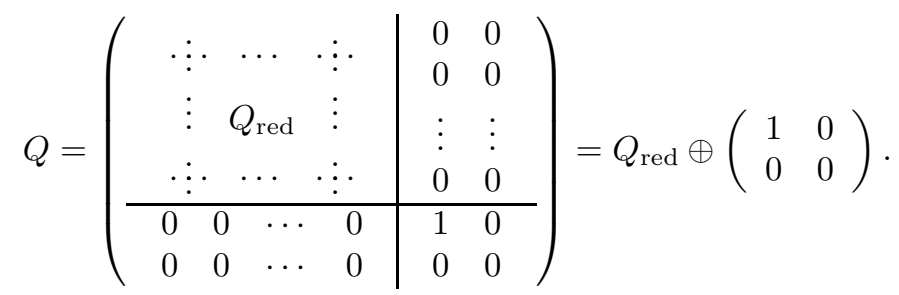


But the case $Q_{0, N-1}=-1$, i.e., $\lambda_{0, N-1}=\lambda_{N-1,0}=1$, cannot occur. To see this, recall that, by (5.24) and (5.49), the $\lambda_{i, j}$ numbers are the matrix entries of $\mathbb{1}_{N}-Q$, which is a projection in $\mathbb{C}^{N}$, since $Q$ is. Specifically,

$$
\lambda_{0, N-1}=-\left\langle\varepsilon_{0} \mid Q \varepsilon_{N-1}\right\rangle,
$$

where $\left\{\varepsilon_{i}\right\}_{i=0}^{N-1}$ is the canonical basis for $\mathbb{C}^{N}$. So we are considering the possibility of having an off-diagonal entry $\lambda_{0, N-1}$ assume the value 1 , and that is impossible. In fact, let $P=P^{*}=P^{2}$ be any projection, and let $\varepsilon, \varepsilon^{\prime}$ be given orthogonal unit vectors. Then

$$
\left|\left\langle\varepsilon \mid P \varepsilon^{\prime}\right\rangle\right| \leq \frac{1}{2}
$$

and so in particular the value 1 is excluded. Note that (5.58) is clearly sharp: take $\left(\begin{array}{ll}1 / 2 & 1 / 2 \\ 1 / 2 & 1 / 2\end{array}\right)$. To prove $(5.58)$, note that

$$
\left|\left\langle\varepsilon \mid P \varepsilon^{\prime}\right\rangle\right|^{2}+\left|\left\langle\varepsilon^{\prime} \mid P \varepsilon^{\prime}\right\rangle\right|^{2} \leq\left\|P \varepsilon^{\prime}\right\|^{2},
$$

by Bessel. Since $\left\langle\varepsilon^{\prime} \mid P \varepsilon^{\prime}\right\rangle=\left\|P \varepsilon^{\prime}\right\|^{2}$, we get

$$
\begin{aligned}
& \left|\left\langle\varepsilon \mid P \varepsilon^{\prime}\right\rangle\right| \leq \sqrt{\left\|P \varepsilon^{\prime}\right\|^{2}-\left\|P \varepsilon^{\prime}\right\|^{4}}=\left\|P \varepsilon^{\prime}\right\| \cdot\left(1-\left\|P \varepsilon^{\prime}\right\|^{2}\right)^{1 / 2} \\
& \quad=\left\|P \varepsilon^{\prime}\right\| \cdot\left\|(\mathbb{1}-P) \varepsilon^{\prime}\right\| \leq \frac{1}{2}\left(\left\|P \varepsilon^{\prime}\right\|^{2}+\left\|(\mathbb{1}-P) \varepsilon^{\prime}\right\|^{2}\right)=\frac{1}{2}\left\|\varepsilon^{\prime}\right\|^{2}=\frac{1}{2} .
\end{aligned}
$$

This proves (5.58).

REMARK 5.8. So in summary, we may define a transformation $\sigma$ for any system of numbers $\lambda_{i, j}$ via (5.50), or the matrix (5.62) below, and its spectrum will be given by (5.51). We are then interested in when points from the spectrum of $\sigma$ can attain the value 1 , and we saw that some can, and others cannot, viz., if the $\lambda_{i, j}$ are derived from an $A$ with $g=2$, then we get the following estimates on the points $\lambda_{0}, \lambda_{N-1}-\lambda_{N-2}, \lambda_{0, N-1}$, and $\lambda_{N-1,0}$ in the spectrum of $\sigma$ :

$$
0 \leq \lambda_{0} \leq 1, \quad-1 \leq \lambda_{N-1}-\lambda_{N-2} \leq 1,
$$

and, by the middle step in (5.60),

$$
\left|\lambda_{0, N-1}\right|=\left|\lambda_{N-1,0}\right| \leq \min \left\{\left(\lambda_{0} \cdot\left(1-\lambda_{0}\right)\right)^{1 / 2},\left(\lambda_{N-1} \cdot\left(1-\lambda_{N-1}\right)\right)^{1 / 2}\right\} \leq \frac{1}{2} .
$$

In particular, the spectral radius of such a $\sigma=\sigma^{(A)}$ is at most 1 . Nonetheless, we know that $\sigma^{(A)}$ is generally not contractive in the Hilbert space $\mathcal{B}\left(\mathcal{K}^{(A)}\right)$ of Hilbert-Schmidt operators, although $\sigma^{(A)}$ obviously is contractive as a completely positive map on the $C^{*}$-algebra $\mathcal{B}\left(\mathcal{K}^{(A)}\right)$.

Remark 5.9. Hence for a given unitary $V \in \mathrm{U}(N)$ and projection $Q \in \mathcal{B}\left(\mathbb{C}^{N}\right)$, we see that the eigenvalue 1 may have the following multiplicities in the characteristic polynomial for various values of $A \sim(V, Q)$ :

1 has multiplicity 3: This occurs in one case. 
CASE 1. $\lambda_{0}=1, \lambda_{N-2}=0$, and $\lambda_{N-1}=1$. The projection $\mathbb{1}-Q$ then has the form

$$
\mathbb{1}-Q=\left(\begin{array}{c|ccc|cc}
1 & 0 & \cdots & 0 & 0 & 0 \\
\hline 0 & \cdots & \cdots & . \vdots & 0 & 0 \\
\vdots & \vdots & P & \vdots & \vdots & \vdots \\
0 & \cdots & \cdots & \cdots & 0 & 0 \\
\hline 0 & 0 & \cdots & 0 & 0 & 0 \\
0 & 0 & \cdots & 0 & 0 & 1
\end{array}\right)
$$

where $P$ is a projection in $\mathcal{B}\left(\mathbb{C}^{N-3}\right)$ (so when $N=3$ there is no more choice.)

1 has multiplicity 2: This occurs in two mutually exclusive cases.

CASE 2. $\lambda_{0}=1$ and $\left(\lambda_{N-2}>0\right.$ or $\left.\lambda_{N-1}<1\right)$.

CASE 3. $\lambda_{0}<1, \lambda_{N-2}=0$, and $\lambda_{N-1}=1$.

1 has multiplicity 1: This occurs in all remaining cases.

Since the dimension of the fixed-point set of $\sigma^{(A)}$ is always at most equal to the multiplicity of 1 in the characteristic polynomial, it follows from Remark 5.9 and Theorem 5.1 that the linear dimension of the commutant of the $T^{(A)}\left(=T^{(V, Q)}\right)$ representation is always at most 3 . Since the smallest-dimensional nonabelian $C^{*}$ algebra is $\mathcal{B}\left(\mathbb{C}^{2}\right)$, which has dimension 4 , it follows that the commutant is always abelian when $g=2$, and the representation decomposes into a sum of at most three irreducible mutually disjoint representations. In order to get the exact number, we have to compute the fixed-point space of $\sigma^{(A)}$ exactly. To this end, we note that the matrix of $\sigma^{(A)}$, relative to the basis $\left\{E_{i, k} ;-2 \leq i, k \leq 0\right\}$ of $\mathcal{B}\left(\mathbb{C}^{3}\right)$ is (we actually compute the matrix of $\sigma^{(A) *}$ using $(5.50)$ and take the adjoint)

\begin{tabular}{r|ccc|ccc|ccc}
$\sigma^{(A)}$ & $(0,0)$ & $(-1,-1)$ & $(-2,-2)$ & $(0,-1)$ & $(0,-2)$ & $(-1,-2)$ & $(-2,-1)$ & $(-1,0)$ & $(-2,0)$ \\
\hline$(0,0)$ & $\lambda_{0}$ & $1-\lambda_{0}$ & 0 & 0 & 0 & 0 & 0 & 0 & 0 \\
$(-1,-1)$ & 0 & $\lambda_{N-1}$ & $1-\lambda_{N-1}$ & 0 & 0 & 0 & 0 & 0 & 0 \\
$(-2,-2)$ & 0 & $\lambda_{N-2}$ & $1-\lambda_{N-2}$ & 0 & 0 & 0 & 0 & 0 & 0 \\
\hline$(0,-1)$ & 0 & 0 & 0 & $\lambda_{N-1,0}$ & 0 & $-\lambda_{N-1,0}$ & 0 & 0 & 0 \\
$(0,-2)$ & 0 & 0 & 0 & $\lambda_{N-2,0}$ & 0 & $-\lambda_{N-2,0}$ & 0 & 0 & 0 \\
$(-1,-2)$ & 0 & $\lambda_{N-2, N-1}$ & $-\lambda_{N-2, N-1}$ & 0 & 0 & 0 & 0 & 0 & 0 \\
\hline$(-2,-1)$ & 0 & $\lambda_{N-1, N-2}$ & $-\lambda_{N-1, N-2}$ & 0 & 0 & 0 & 0 & 0 & 0 \\
$(-1,0)$ & 0 & 0 & 0 & 0 & 0 & 0 & $-\lambda_{0, N-1}$ & $\lambda_{0, N-1}$ & 0 \\
$(-2,0)$ & 0 & 0 & 0 & 0 & 0 & 0 & $-\lambda_{0, N-2}$ & $\lambda_{0, N-2}$ & 0 \\
\hline
\end{tabular}

Thus one may compute the eigenvectors of $\sigma^{(A)}$. The generic result is as in Table 1.

Note that in non-generic cases, like $\lambda_{0}=1$, and/or $\left(\lambda_{N-1}=1\right.$ and $\left.\lambda_{N-2}=0\right)$, some of the listed eigenvectors are zero. This is not surprising since these are exactly the cases where the root 1 in the characteristic polynomial is multiple. Let us consider these cases separately: 
TABLE 1. Eigenvalues, multiplicities, and eigenvectors of $\sigma^{(A)}$

\begin{tabular}{l|l|l} 
Multiplicity & Eigenvalue & Eigenvectors \\
\hline 4 & 0 & $E_{0,-2}, E_{-2,0}, E_{0,-1}+E_{-1,-2}, E_{-1,0}+E_{-2,-1}$ \\
\hline 1 & $\lambda_{0}$ & $E_{0,0}$ \\
\hline 1 & 1 & $\mathbb{1}_{\mathcal{K}}=E_{0,0}+E_{-1,-1}+E_{-2,-2}$ \\
\hline 1 & $\lambda_{N-1}-\lambda_{N-2}$ & $\begin{array}{r}\left(1-\lambda_{0}\right)\left(1-\lambda_{N-1}\right) E_{0,0} \\
+\left(\lambda_{N-1}-\lambda_{N-2}-\lambda_{0}\right)\left(1-\lambda_{N-1}\right) E_{-1,-1} \\
-\lambda_{N-2}\left(\lambda_{N-1}-\lambda_{N-2}-\lambda_{0}\right) E_{-2,-2}\end{array}$ \\
\hline 1 & $\lambda_{N-1,0}$ & $\lambda_{N-1,0} E_{0,-1}+\lambda_{N-2,0} E_{0,-2}$ \\
\hline 1 & $\lambda_{0, N-1}=\overline{\lambda_{N-1,0}}$ & $\lambda_{0, N-1} E_{-1,0}+\lambda_{0, N-2} E_{-2,0}$ \\
\hline
\end{tabular}

CASE 2. $\lambda_{0}=1\left(\Rightarrow \lambda_{0, N-1}=\lambda_{0, N-2}=0\right)$ and $\lambda_{N-1}<1$ or $\lambda_{N-2}>0$ : In this case the last two eigenvectors in the list in Table 1 are zero, but one may verify that these may be replaced by the vectors $E_{0,-2}$ and $E_{-2,0}$. Thus the eigenspace corresponding to 1 is indeed 2 -dimensional in this case, and spanned by $\mathbb{1}_{\mathcal{K}}$ and $E_{0,0}$.

CASE 3. $\lambda_{0}<1, \lambda_{N-1}=1, \lambda_{N-2}=0$ : In this case it follows from (5.54) that $\lambda_{N-1, k}=0$ for all $k \neq N-1$ and $\lambda_{N-2, k}=0$ for all $k \neq N-2$, so the matrix in (5.62) degenerates into the upper-left-hand $3 \times 3$ matrix, which is

$$
\left(\begin{array}{ccc}
\lambda_{0} & 1-\lambda_{0} & 0 \\
0 & 1 & 0 \\
0 & 0 & 1
\end{array}\right) .
$$

So we see that a new eigenvector for $\lambda_{N-1}-\lambda_{N-2}=1$ is

$$
E_{-2,-2} \text {. }
$$

Let us now look at the even more degenerate case where the multiplicity of 1 in the characteristic polynomial is 3 :

CASE 1. $\lambda_{0}=1, \lambda_{N-1}=1, \lambda_{N-2}=0$ : From the discussion of Case 3 above, it follows that then the upper-left $3 \times 3$ matrix of (5.62) is $\mathbb{1}_{3}$, while the rest of the matrix is zero. Thus the fixed-point set is the linear span of

$$
E_{0,0}, E_{-1,-1} \text {, and } E_{-2,-2} \text {. }
$$

Thus the dimension of the eigensubspace is in all cases where the parameters are restricted as in Remark 5.8 equal to the multiplicity of the root 1 in the characteristic polynomial, and the above analysis implies

Corollary 5.10. If $g=2$ and $N>2$, the representation $T^{(A)}$ decomposes into at most three irreducible representations which are mutually nonequivalent. Generically, $T^{(A)}$ is irreducible, and otherwise the decomposition structure is summarized in Table 2.

REMARK 5.11. Table 2 shows a remarkable feature which these representations share with the $g=2, N=2$ representations studied in [BEJ99], namely that the 
TABLE 2. Decompositions from eigenvalue-one multiplicities, and eigenvectors for $\sigma^{(A)}$

\begin{tabular}{l|c|l} 
Case & $\begin{array}{l}\text { Number of } \\
\text { subrepresen- } \\
\text { tations }\end{array}$ & $\begin{array}{l}\text { The fixed-point set of } \sigma^{(A)} \\
\text { is spanned by }\end{array}$ \\
\hline $\begin{array}{c}\lambda_{0}=1, \lambda_{N-2}=0, \\
\text { and } \lambda_{N-1}=1\end{array}$ & 3 & $E_{0,0}, E_{-1,-1}$, and $E_{-2,-2}$ \\
\hline $\begin{array}{c}\lambda_{0}<1, \lambda_{N-1}=1, \\
\text { and } \lambda_{N-2}=0\end{array}$ & 2 & $\begin{array}{l}\mathbb{1}_{K} \text { and } E_{-2,-2} \\
\left.\text { (i.e., } E_{0,0}+E_{-1,-1} \text { and } E_{-2,-2}\right)\end{array}$ \\
\hline $\begin{array}{c}\lambda_{0}=1 \text { and } \\
\left(\lambda_{N-1}<1 \text { or } \lambda_{N-2}>0\right)\end{array}$ & 2 & $\begin{array}{l}\mathbb{1}_{K} \text { and } E_{0,0} \\
\left.\text { (i.e., } E_{0,0} \text { and } E_{-1,-1}+E_{-2,-2}\right)\end{array}$ \\
\hline
\end{tabular}

fixed-point set of $\sigma^{(A)}$ is both abelian and an algebra in all cases. Hence it follows from (5.12) that the projection $P$ onto $\mathcal{K}$ commutes with the commutant $\pi\left(\mathcal{O}_{N}\right)^{\prime}$, and thus we may find cyclic vectors for the various subrepresentations by picking vectors in the range of the minimal projections in the fixed-point set $\mathcal{B}(\mathcal{K})^{\sigma^{(A)}}$. For example, for the first case in Table 2 (Case 1), the three vectors $z^{-n}, n=0,1,2$, in $L^{2}(\mathbb{T})$ are cyclic for the three disjoint representations the original representation decomposes into, respectively. For the second case in Table 2 (Case 3), the pair $z^{0}, z^{-2}$ has the same property, as well as the pair $z^{-1}, z^{-2}$ or any pair of the form $\lambda z^{0}+\mu z^{-1}, z^{-2}$. So far we do not know a single example (for general $N, g$ ) where $\mathcal{B}(\mathcal{K})^{\sigma^{(A)}}$ is not abelian, or is not an algebra.

\section{Reduction of representations as a reduction of $N$}

Consider an arbitrary element $A \in \mathcal{P}(\mathbb{T}, \mathrm{U}(N))$. Hence both $N$ and the genus $g$ are arbitrary, but given. We saw that there is an associated wavelet filter $m^{(A)}$, as well as a representation $T^{(A)}$ of $\mathcal{O}_{N}$ acting on $L^{2}(\mathbb{T})$. (As before we work in the standard Fourier basis for $L^{2}(\mathbb{T})$, i.e., $e_{n}(z)=z^{n}, n \in \mathbb{Z}$.) We identified the subspace $\mathcal{K}$ spanned by $\left\{e_{-r}, \ldots, e_{-1}, e_{0}\right\}$ where $r$ was picked so that reducibility of $T^{(A)}$ is decided by the fixed-point set of an associated completely positive map $\sigma_{\mathcal{K}}^{(A)}$ given by

$$
\sigma_{\mathcal{K}}^{(A)}(\cdot)=\sum_{i=0}^{N-1} V_{i}^{(A)}(\cdot) V_{i}^{(A) *}
$$

with

$$
V_{i}^{(A)}=\mathcal{P}_{\mathcal{K}} T_{i}^{(A)}
$$

While we identified the complete spectral picture of $\sigma_{\mathcal{K}}^{(A)}$ in the special case $g=2$, the higher-genus case is not yet entirely understood. But we note that the identity from the $g=2$ case,

$$
\sigma_{\mathcal{K}}^{(A)}\left(E_{0,0}\right)=\lambda_{0}(A) E_{0,0},
$$

which is clear from (5.62), remains true for arbitrary $g$. Hence our use of $\sigma_{\mathcal{K}}^{(A)}$ yields reducibility of the representation $T^{(A)}$ whenever $\lambda_{0}(A)=1$. We recall that if

$$
A(z)=A^{(0)}+z A^{(1)}+\cdots+z^{g-1} A^{(g-1)},
$$


then $\lambda_{0}(A)$ is defined as the $(0,0)$-matrix entry in $A^{(0) *} A^{(0)}$.

REMARK 6.1. To prove (6.3) for the general case, recall that

$$
T_{i}^{(A)} e_{0}=\sum_{j=0}^{N-1} A_{i, j}\left(z^{N}\right) e_{j}=\sum_{j=0}^{N-1} \sum_{k=0}^{g-1} A_{i, j}^{(k)} e_{j+k N} .
$$

Using (6.2), we therefore get

$$
V_{i}^{(A)} e_{0}=P_{\mathcal{K}} T_{i}^{(A)} e_{0}=\sum_{j=0}^{N-1} \sum_{k=0}^{g-1} A_{i, j}^{(k)} P_{\mathcal{K}} e_{j+k N}=A_{i, 0}^{(0)} e_{0},
$$

and by (5.45),

$$
\begin{aligned}
\sigma_{\mathcal{K}}^{(A)}\left(E_{0,0}\right) & =\left(\sum_{i=0}^{N-1}\left|A_{i, 0}^{(0)} e_{0}\right\rangle\left\langle A_{i, 0}^{(0)} e_{0}\right|\right)=\left(\sum_{i=0}^{N-1}\left|A_{i, 0}^{(0)}\right|^{2}\right)\left|e_{0}\right\rangle\left\langle e_{0}\right| \\
& =\left(A^{(0) *} A^{(0)}\right)_{0,0} E_{0,0}=\lambda_{0}(A) E_{0,0},
\end{aligned}
$$

which is 6.3 .

But it is worth stressing that the argument (6.6), for the vector $e_{0}$ in $\mathcal{K}$, does not carry over to the other basis vectors $e_{-1}, e_{-2}, \ldots$ This is clear already in the case $g=2$ from (5.62). More generally, for $e_{-1}$, for example,

$$
V_{i}^{(A)} e_{-1}=A_{i, 0}^{(1)} e_{0}+A_{i, N-1}^{(0)} e_{-1}+A_{i, N-2}^{(0)} e_{-2}+\cdots,
$$

and therefore

$$
\begin{aligned}
& \sigma_{\mathcal{K}}^{(A)}\left(E_{-1,-1}\right)=\lambda_{0,0}^{(11)} E_{0,0}+\lambda_{N-1, N-1}^{(00)} E_{-1,-1}+\lambda_{N-2, N-2}^{(00)} E_{-2,-2}+\cdots \\
+ & \lambda_{N-1,0}^{(01)} E_{0,-1}+\lambda_{0, N-1}^{(10)} E_{-1,0}+\lambda_{N-2, N-1}^{(00)} E_{-1,-2}+\lambda_{N-1, N-2}^{(00)} E_{-2,-1}+\cdots
\end{aligned}
$$

where $\lambda_{i, j}^{(k l)}:=\left(A^{(k) *} A^{(l)}\right)_{i, j}$, i.e., the $(i, j)$ entry in the $N \times N$ matrix $A^{(k) *} A^{(l)}$. Even if $\lambda_{N-1, N-1}^{(00)}=1$, that does not imply that all the other coefficients $\lambda_{i, j}^{(k l)}$ from (6.9) will necessarily vanish; see the details below. While

$$
\lambda_{N-1, N-1}^{(00)}=1 \Longrightarrow \lambda_{N-1, j}^{(00)}=\lambda_{i, N-1}^{(00)}=0 \quad \text { for all } i, j \in\{0, \ldots, N-2\},
$$

the other coefficients in (6.9) such as $\lambda_{N-2, N-2}^{(00)}$ or $\lambda_{0,0}^{(11)}$ would typically be nonzero even if $\lambda_{N-1, N-1}^{(00)}=1$. So even then, $E_{-1,-1}$ will not be fixed by $\sigma_{\mathcal{K}}^{(A)}$, and there is not a natural analogue to 6.3$)$. While if $g=2$, then the difference $\lambda_{N-1, N-1}^{(00)}$ $\lambda_{N-2, N-2}^{(00)}$ is in the spectrum of $\sigma_{\mathcal{K}}^{(A)}$, see Table 1, this will generally not be true if $g>2$. Then the spectral picture for $\sigma_{\mathcal{K}}^{(A)}$ is not fully understood.

However, the following result shows that a general wavelet representation $T^{(A)}$ may be reducible because a special point $\lambda_{0}(A)$ in the spectrum of $\sigma_{\mathcal{K}}^{(A)}$ may be one. This is a special reduction, however, as we also showed that whenever any point $\lambda$ in spectrum $\left(\sigma_{\mathcal{K}}^{(A)}\right)$ satisfies $\lambda=1$, we get a possibly different reduction of the representation $\left(T^{(A)}, L^{2}(\mathbb{T})\right)$.

Theorem 6.2. Let $A \in \mathcal{P}(\mathbb{T}, \mathrm{U}(N))$. Then the following five conditions are equivalent.

(i) $\lambda_{0}(A)=1$ 
(ii) There is a $B=\left(B_{i, j}\right)_{i, j=1}^{N-1} \in \mathcal{P}(\mathbb{T}, \mathrm{U}(N-1))$ such that

$$
A(1)^{-1} A(z)=\left(\begin{array}{c|cccc}
1 & 0 & 0 & \cdots & 0 \\
\hline 0 & B_{1,1}(z) & \cdots & \cdots & B_{1, N-1}(z) \\
0 & \vdots & & & \vdots \\
\vdots & \vdots & & & \vdots \\
0 & B_{N-1,1}(z) & \cdots & \cdots & B_{N-1, N-1}(z)
\end{array}\right),
$$

i.e.,

$$
A(1)^{-1} A(z)=\left(\begin{array}{c|ccc}
1 & 0 & \cdots & 0 \\
\hline 0 & & & \\
\vdots & & B(z) & \\
0 & & &
\end{array}\right)=(1) \oplus B(z) .
$$

(iii) After modifying with a $\mathrm{U}(N)$-automorphism of $\mathcal{O}_{N}$, the corresponding wavelet filter $m^{(A)}$ satisfies

$$
\left\{\begin{array}{l}
m_{0}^{(A)}(z) \equiv 1, \text { for all } z \in \mathbb{T} \\
m_{i}^{(A)}(z)=\sum_{j=1}^{N-1} B_{i, j}\left(z^{N}\right) z^{j} .
\end{array}\right.
$$

$$
T_{i}^{(A)^{*}} e_{0} \in \mathbb{C} e_{0}, \text { for all } i=0, \ldots, N-1 \text {. }
$$

$(\mathrm{v})$

$$
\sigma_{\mathcal{K}}^{(A) *}\left(E_{0,0}\right) \in \mathbb{C} E_{0,0}
$$

REMARK 6.3. Formula (6.13) explains the assertion made in the Introduction about reduction in the size of the number $N$ which serves as the scaling of the wavelet in question. Once $B$ is identified then there is an $(N-1)$-wavelet filter

$$
m_{i-1}^{(B)}(z)=\sum_{j=0}^{N-2} B_{i, j+1}\left(z^{N-1}\right) z^{j},
$$

$i=1, \ldots, N-1$.

Proof. (还 $\Rightarrow$ (目): Using the factorization $(5.22)$ in Corollary 5.2, we note that

$$
\begin{aligned}
A^{(0) *} A^{(0)}=\left(\mathbb{1}-Q_{g-1}\right)\left(\mathbb{1}-Q_{g-2}\right) \cdots & \\
& \cdots\left(\mathbb{1}-Q_{1}\right)\left(\mathbb{1}-Q_{0}\right)\left(\mathbb{1}-Q_{1}\right) \cdots \\
\cdots\left(\mathbb{1}-Q_{g-2}\right)\left(\mathbb{1}-Q_{g-1}\right) &
\end{aligned}
$$

and the $V(\in \mathrm{U}(N))$ from (5.22) is $V=A(1)$, i.e., evaluation of $A(z)$ at $z=1$. Let $\varepsilon_{0}$ be the first canonical basis vector in $\mathbb{C}^{N}$. Then (ii) states that

$$
\left\|\left(\mathbb{1}-Q_{0}\right)\left(\mathbb{1}-Q_{1}\right) \cdots\left(\mathbb{1}-Q_{g-1}\right) \varepsilon_{0}\right\|=1 .
$$

Hence $\left\langle\left(\mathbb{1}-Q_{g-1}\right) \varepsilon_{0} \mid H\left(\mathbb{1}-Q_{g-1}\right) \varepsilon_{0}\right\rangle=1$, where

$$
H:=\left(\mathbb{1}-Q_{g-2}\right) \cdots\left(\mathbb{1}-Q_{1}\right)\left(\mathbb{1}-Q_{0}\right)\left(\mathbb{1}-Q_{1}\right) \cdots\left(\mathbb{1}-Q_{g-2}\right) .
$$


Using Schwarz's inequality and induction, we conclude that $\left(\mathbb{1}_{N}-Q_{j}\right) \varepsilon_{0}=\varepsilon_{0}$, and therefore $Q_{j} \varepsilon_{0}=0$ for all $j=0, \ldots, g-1$. Hence $\left(\mathbb{1}_{N}-Q_{j}+z Q_{j}\right) \varepsilon_{0}=\varepsilon_{0}$, and from (5.22),

$$
A(1)^{-1} A(z) \varepsilon_{0}=\varepsilon_{0} .
$$

The same argument yields

$$
\left\langle\varepsilon_{0} \mid A(1)^{-1} A(z) e_{j}\right\rangle=\delta_{0, j},
$$

and so (iii) follows.

(iii) $\Rightarrow$ (iii): Substituting (6.11) into (2.24), we get

$$
\begin{aligned}
& \left(V^{-1} A\right)_{i, 0}(z)=\delta_{i, 0}, \\
& \left(V^{-1} A\right)_{0, j}(z)=\delta_{j, 0},
\end{aligned}
$$

and

$$
\left(V^{-1} A\right)_{i, j}(z)=B_{i, j}(z) \quad \text { if } i, j \geq 1,
$$

and (6.13) in (iii) follows.

(iii) $\Rightarrow$ (iv): Using

$$
T_{i}^{(A) *} \xi(z)=\frac{1}{N} \sum_{w^{N}=z} \overline{m_{i}^{(A)}(w)} \xi(w), \quad \xi \in L^{2}(\mathbb{T}),
$$

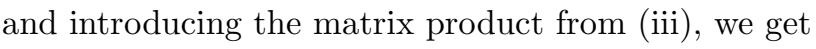

$$
\left\{\begin{array}{l}
A_{i, 0}(z)=V_{i, 0}, \\
A_{i, j}(z)=\sum_{k=1}^{n-1} V_{i, k} B_{i, k}(z), j>0 .
\end{array}\right.
$$

Using again (2.24), we thus get

$$
T_{i}^{(A) *} e_{0}=\overline{V_{i, 0}} e_{0},
$$

where $V_{i, j}$ denotes the matrix entries of $V(:=A(1))$ in $\mathrm{U}(N)$.

(iiv) $\Leftrightarrow($ 冈 $) \Rightarrow($ i $):$ By $(6.23)$,

$$
T_{i}^{(A) *} e_{0}=\overline{A_{i, 0}(z)} \in \mathcal{P}(\mathbb{T}, \mathbb{C}) ;
$$

and so if (iv) is assumed, then

$$
A_{i, 0}^{(k)}=0 \quad \text { for } k>0 .
$$

Since

$$
\sum_{i} \overline{A_{i, 0}(z)} A_{i, 0}(z)=1
$$

in general, we get

$$
\lambda_{0}(A)=\sum_{i} \overline{A_{i, 0}^{(0)}} A_{i, 0}^{(0)}=1 .
$$

From $(6.26)$, we get

$$
T_{i}^{(A) *} e_{0}=\sum_{k=0}^{g-1} \overline{A_{i, 0}^{(k)}} e_{-k},
$$


and by $(5.46)$,

$$
\sigma_{\mathcal{K}}^{(A) *}\left(E_{0,0}\right)=\sum_{k=0}^{g-1} \sum_{l=0}^{g-1} \lambda_{0,0}^{(k l)} E_{-k,-l} .
$$

Note the coefficient of $E_{0,0}$ in (6.31) is $\lambda_{0}(A)=\lambda_{0,0}^{(00)}$. For the other coefficients, we have

$$
\left|\lambda_{0,0}^{(k l)}\right|^{2} \leq \lambda_{0,0}^{(k k)} \lambda_{0,0}^{(l l)}
$$

and

$$
\lambda_{0,0}^{(k k)}=\sum_{i=0}^{N-1}\left|A_{i, 0}^{(k)}\right|^{2} .
$$

Hence, the vanishing of the non- $(0,0)$ coefficients in (6.31) is equivalent to condition (6.27), which we already showed is equivalent to both (iv) and (ii).

For $\xi \in \mathcal{K}$, let $\mathcal{H}_{+}^{(A)}[\xi]$ denote the cyclic subspace in $L^{2}(\mathbb{T})$ generated by $\xi$ and the operators $T_{i}^{(A)}$, and let $E_{+}^{(A)}[\xi]$ be the corresponding projection.

Corollary 6.4. Suppose condition (1) of Theorem 6.2 holds. Then the (unique) operator $W$ in the commutant of $T^{(A)}$ which satisfies

$$
P_{\mathcal{K}} W P_{\mathcal{K}}=E_{0,0}
$$

is $W=E_{+}^{(A)}\left[e_{0}\right]$.

Proof. Let $v \in \mathbb{C}^{N}$ be the vector with coordinates $v_{i}:=A_{i, 0}^{(0)}$. Then (i) holds if and only if $\|v\|=1$. In that case, the restriction of $T^{(A)}$ to $\mathcal{H}_{+}^{(A)}\left[e_{0}\right]$ is the subrepresentation which is induced by the Cuntz state Cun77 $\omega_{v}$. Specifically, if $I=\left(i_{1}, \ldots, i_{k}\right), J=\left(j_{1}, \ldots, j_{l}\right)$ are multi-indices, then it follows from (iiv) that

$$
\left\langle e_{0} \mid T_{I}^{(A)} T_{J}^{(A) *} e_{0}\right\rangle=v_{i_{1}} \cdots v_{i_{k}} \overline{{j_{1}}_{1}} \cdots \overline{v_{j_{l}}}=\omega_{v}\left(s_{I} s_{J}^{*}\right) .
$$

Hence $\mathcal{H}_{+}^{(A)}\left[e_{0}\right]$ is the closed subspace spanned by $e_{0}$ and $\left\{T_{I}^{(A)} e_{0}\right\}$. Since

$$
T_{i}^{(A)} e_{0}(z)=\sum_{j=0}^{N-1} A_{i, j}\left(z^{N}\right) z^{j} \in \mathcal{P}(\mathbb{T}, \mathbb{C}),
$$

$\mathcal{H}_{+}^{(A)}\left[e_{0}\right]$ is contained in the Hardy space $\mathcal{H}_{+}:=\overline{\operatorname{span}}\left\{e_{n} ; n \geq 0\right\}$. Since $\mathcal{K}=$ $\operatorname{span}\left\{e_{-r}, \ldots, e_{-1}, e_{0}\right\}$, it follows that $E_{+}^{(A)}\left[e_{0}\right]$ satisfies $(6.34)$. Since $\sigma^{(A)}\left(E_{0,0}\right)=$ $E_{0,0}$, the operator $W$ in the commutant of $T^{(A)}$ satisfying (6.34) is unique by Theorem 5.1, and so $W=E_{+}^{(A)}\left[e_{0}\right]$.

REMARK 6.5. The Hilbert space prior to Corollary 6.4 is of the form $\mathcal{H}_{+}^{(A)}[\xi]$ for some $\xi \in \mathcal{K}$ where

$$
T_{i}^{(A) *} \xi=v_{i} \xi
$$

for some $v=\left(v_{i}\right) \in \mathbb{C}^{N}$. Since $\omega_{v}$ is a Cuntz state, the corresponding representation is unique from $v$ up to unitary equivalence, by [Cun77]. It also follows from Jor99, Theorem 6.3] that every $\xi \in \mathcal{K}$ which satisfies (6.37) for some $v \in \mathbb{C}^{N}$ must be a 
monomial, i.e., $\xi(z)=z^{-k}$ for some $k \in\{0,1, \ldots, r\}$. In our special setting, this can be proved as follows: If $T_{i}^{*} \xi=\bar{v}_{i} \xi$ for a unit vector $\xi \in \mathcal{K}$, where $\sum_{i}\left|v_{i}\right|^{2}=1$, then $\xi=\sum_{i} T_{i} T_{i}^{*} \xi=\sum_{i} \bar{v}_{i} T_{i} \xi$, or, spelled out, $\xi(z)=\sum_{i} \bar{v}_{i} m_{i}(z) \xi\left(z^{N}\right)$. Hence, putting $m(z)=\sum_{i} \bar{v}_{i} m_{i}(z)$, we have $\xi(z)=m(z) \xi\left(z^{N}\right)$. Now we may use the argument from Jor99, page 104] or BrJo97b, Theorem 3.1] to conclude that $|m(z)|=1$ and $|\xi(z)|=1$ for almost all $z \in \mathbb{T}$. But both $m(z)$ and $\overline{\xi(z)}$ are polynomials, and we deduce from Lemma 3.1 that they are monomials. If $\xi(z)=z^{-k}$ it follows that $m(z)=\xi(z) \overline{\xi\left(z^{N}\right)}=z^{(N-1) k}$. Thus a very restrictive necessary and sufficient condition for the Cuntz state $\omega_{v}$ to occur is that

$$
\sum_{i} \bar{v}_{i} m_{i}(z)=z^{(N-1) k} \quad \text { for some } k \in\{0,1, \ldots, r\} .
$$

The other details of the decompositions may be spelled out as follows: Let $\mathcal{H}=L^{2}(\mathbb{T})$. Note first that Corollary 6.4 holds whenever $E_{0,0}$ is replaced by any one-dimensional projection in the fixed-point set of $\sigma$ in the generality of Theorem 5.1. since $E_{0,0}(\mathcal{K})=E_{0,0}(\mathcal{H})$ is then a one-dimensional $T^{(A)}{ }^{*}$-invariant space. If $e$ is a general (not necessarily one-dimensional) projection in the fixed-point set, it follows from Lemma 3.3 in BJKW97 that $e$ commutes with all the operators $P T_{i}^{(A){ }^{*} P}$ and $P T_{i}^{(A)} P$ and hence the projection $E$ onto $\left[\mathcal{O}_{N} e \mathcal{K}\right]$ in $\mathcal{H}$ is in the commutant of the representation, and $P E P=e$. This justifies claims in Remark 5.11. In the case $g=2$, which is completely enumerated in BEJ99 (for $N=2$ ) and in Table 2 (for $N>2$ ), the ranges of the projections in the fixed-point set are spanned by subsets of the orthonormal set $\left\{e_{0}, e_{-1}, \ldots, e_{-r}\right\}$, and hence each Hilbert space in the decomposition has the form $E_{+}^{(A)}[\xi]$ for a suitable $\xi$ in this set. The vector $\xi$ defines a Cuntz state if and only if the corresponding projection in $\mathcal{B}(\mathcal{K})^{\sigma}$ is one-dimensional. Note also that the result [Jor99, Theorem 6.3] spelled out above implies that all one-dimensional projections in $\mathcal{B}(\mathcal{K})^{\sigma}$ are diagonal in the standard basis. This gives a partial explanation of these results.

Other examples of decompositions of $L^{2}(\mathbb{T})$. Generically, the wavelet examples do not have minimal projections $e$ in $\mathcal{B}(\mathcal{K})^{\sigma}$ of dimension one, and even if there is one such projection in $\mathcal{B}(\mathcal{K})^{\sigma}$, there may be others that do not have dimension one. For illustration, let us repeat some of the examples in [BEJ99], rephrased in the setting of the present paper. Take, for example, $N=2=g$. Then, by (6.38), only the two cases $\left(\begin{array}{ll}1 & 0 \\ 0 & z\end{array}\right)$ and $\left(\begin{array}{ll}z & 0 \\ 0 & 1\end{array}\right)$ have the Cuntz-state vectors. For $A(z)=\left(\begin{array}{ll}1 & 0 \\ 0 & z\end{array}\right)$, even though $\lambda_{0}(A)=1$, this example has a minimal 2-dimensional projection $e$ in $\mathcal{B}(\mathcal{K})^{\sigma}$, namely $e=E_{-1,-1}+E_{-2,-2}$. It is minimal in the sense that the restriction of $T^{(A)}$ to $E_{e}=\left[\mathcal{O}_{2} e \mathcal{K}\right]$ is irreducible; see [BEJ99, (4.52)]. The matrix $A(z)=\left(\begin{array}{ll}0 & 1 \\ z & 0\end{array}\right)$ has $\lambda_{0}(A)=0$, and its representation $T^{(A)}$ decomposes into a sum of two irreducibles associated with respective 2-dimensional projections $e$ and $f$ in $\mathcal{B}(\mathcal{K})^{\sigma}: e=E_{0,0}+E_{-1,-1}$ and $f=E_{-2,-2}+E_{-3,-3}$; see BEJ99, (4.46)]. Hence in this case, $\mathcal{H}=L^{2}(\mathbb{T})=\left[\mathcal{O}_{2} e \mathcal{K}\right] \oplus\left[\mathcal{O}_{2} f \mathcal{K}\right]$ with $T^{(A)}$ restricting to an irreducible representation on each of the two subspaces. A direct application of (5.14 - 5.15) in Theorem 5.1 shows that these two subrepresentations are disjoint, i.e., inequivalent. While these examples have $g=2$, the cases $g>2$ entail a richer decomposition structure. 
Note that the result, Theorem 5.1, which is used in analyzing the representations $T^{(A)}$, applies also when $g>2$. Moreover, the factorization result, Corollary 5.2. yields in principle a way of understanding the general case, i.e., arbitrary $g$ and $N$, but unpublished experimentation with examples for $g=3$ (i.e., two projections in $\mathbb{C}^{N}$ ) has not so far yielded decomposition structures more general than the above mentioned ones.

REMARK 6.6. The proof of (6.3) shows more generally that

$$
\sigma^{(B, A)}\left(E_{0,0}\right)=\left(\sum_{i=0}^{N-1} \overline{A_{i, 0}^{(0)}} B_{i, 0}^{(0)}\right) E_{0,0} .
$$

Referring to Theorem 5.1, we note then that the necessary and sufficient condition for $E_{0,0}$ to induce an operator in $L^{2}(\mathbb{T})$ which intertwines the two representations $T^{(A)}$ and $T^{(B)}$ is $\left(A^{(0) *} B^{(0)}\right)_{0,0}=1$. Moreover, if this holds, then both $T^{(A)}$ and $T^{(B)}$ must satisfy the equivalent conditions in Theorem 6.2, and we must then further have $A_{i, 0}^{(0)}=B_{i, 0}^{(0)}$ for all $i$. Then the intertwining operator $W$ which is induced by $E_{0,0}$ via $\sigma^{(B, A)}\left(E_{0,0}\right)=E_{0,0}$ is the one which results from the uniqueness of the $\omega_{v}$-representation where $v_{i}=A_{i, 0}^{(0)}=B_{i, 0}^{(0)}$.

ACKnowledgements. This research was done when one of us (P.E.T.J.) visited the University of Oslo with support from the university and NFR. He is grateful for kind hospitality. We wish to thank Brian Treadway for his skillful typesetting, and Rune Kleveland and Brian Treadway for suggestions and elimination of mistakes in earlier versions of this paper.

This is an expanded version of the invited lecture delivered by P.E.T.J. at the International Conference on Wavelet Analysis and Its Applications held at Zhongshan University, Guangzhou, China, in November of 1999 (the conference announcement may be seen in The Wavelet Digest 8 (1999), on the World Wide Web at http://www.wavelet.org/wavelet/digest_08/digest_08.04.html\#13).

\section{References}

[Ada62] J.F. Adams, Vector fields on spheres, Ann. of Math. (2) 75 (1962), 603-632.

[Bla86] B. Blackadar, K-theory for Operator Algebras, MSRI Publication Series, vol. 5, Springer-Verlag, New York-Heidelberg-Berlin-Tokyo, 1986.

[BEJ99] O. Bratteli, D.E. Evans, and P.E.T. Jorgensen, Compactly supported wavelets and representations of the Cuntz relations, to appear in Appl. Comput. Harmon. Anal. 8 (2000), no. 2. math.FA/9912129

[BrJo97a] O. Bratteli and P.E.T. Jorgensen, Endomorphisms of $\mathcal{B}(\mathcal{H})$, II: Finitely correlated states on $\mathcal{O}_{N}$, J. Funct. Anal. 145 (1997), 323-373.

[BrJo97b] O. Bratteli and P.E.T. Jorgensen, Isometries, shifts, Cuntz algebras and multiresolution wavelet analysis of scale $N$, Integral Equations Operator Theory 28 (1997), 382-443. funct-an/9612003

[BrJo98] O. Bratteli and P.E.T. Jorgensen, A connection between multiresolution wavelet theory of scale $N$ and representations of the Cuntz algebra $\mathcal{O}_{N}$, Operator Algebras and Quantum Field Theory (Rome, 1996) (S. Doplicher, R. Longo, J.E. Roberts, and L. Zsido, eds.), International Press, 1998, pp. 151-155. funct-an/9612006

[BrJo99] O. Bratteli and P.E.T. Jorgensen, Convergence of the cascade algorithm at irregular scaling functions, The Functional and Harmonic Analysis of Wavelets and Frames (San Antonio, 1999) (L.W. Baggett and D.R. Larson, eds.), Contemp. Math., vol. 247, American Mathematical Society, Providence, 1999, pp. 93-130. math.FA/9912133

[BJKW97] O. Bratteli, P.E.T. Jorgensen, A. Kishimoto, and R. Werner, Pure states on $\mathcal{O}_{d}$, J. Operator Theory, to appear. funct-an/9711004 
[BJP96] O. Bratteli, P.E.T. Jorgensen, and G.L. Price, Endomorphisms of $\mathcal{B}(\mathcal{H})$, Quantization, nonlinear partial differential equations, and operator algebra (W. Arveson, T. Branson, and I. Segal, eds.), Proc. Sympos. Pure Math., vol. 59, American Mathematical Society, Providence, 1996, pp. 93-138. funct-an/9408001

[Cun77] J. Cuntz, Simple $C^{*}$-algebras generated by isometries, Comm. Math. Phys. 57 (1977), 173-185.

[Cun80] J. Cuntz, Automorphisms of certain simple $C^{*}$-algebras, Quantum fields-algebras, processes (Bielefeld, 1978) (L. Streit, ed.), Springer-Verlag, Vienna-New York, 1980, pp. 187-196.

[Dau92] I. Daubechies, Ten Lectures on Wavelets, CBMS-NSF Regional Conf. Ser. in Appl. Math., vol. 61, Society for Industrial and Applied Mathematics, Philadelphia, 1992.

[DoRo87] S. Doplicher and J.E. Roberts, Duals of compact Lie groups realized in the Cuntz algebras and their actions on $C^{*}$-algebras, J. Funct. Anal. 74 (1987), 96-120.

[DoRo89] S. Doplicher and J.E. Roberts, Endomorphisms of $C^{*}$-algebras, cross products and duality for compact groups, Ann. of Math. (2) 130 (1989), 75-119.

[GrMa92] K. Gröchenig and W.R. Madych, Multiresolution analysis, Haar bases, and self-similar tilings of $\mathbf{R}^{n}$, IEEE Trans. Inform. Theory 38 (1992), 556-568.

[Hör95] L. Hörmander, Lectures on harmonic analysis, Dept. of Mathematics, Box 118, S22100 Lund, 1995.

[Jor99] P.E.T. Jorgensen, Harmonic analysis of fractal processes via $C^{*}$-algebras, Math. Nachr. 200 (1999), 77-117.

[MRV96] P.R. Massopust, D.K. Ruch, and P.J. Van Fleet, On the support properties of scaling vectors, Appl. Comput. Harmon. Anal. 3 (1996), 229-238.

[Mey87] Y. Meyer, Ondelettes, fonctions splines et analyses graduées, Rend. Sem. Mat. Univ. Politec. Torino 45 (1987), 1-42.

[Pol90] D. Pollen, $\mathrm{SU}_{I}(2, F[z, 1 / z])$ for $F$ a subfield of C, J. Amer. Math. Soc. 3 (1990), 611-624.

[Pol92] D. Pollen, Daubechies' scaling function on [0,3], Wavelets: A Tutorial in Theory and Applications (C.K. Chui, ed.), Wavelet Anal. Appl., vol. 2, Academic Press, Boston, 1992, pp. 3-13.

[PrSe86] A. Pressley and G. Segal, Loop Groups, Oxford mathematical monographs, Oxford science publications, Clarendon Press, Oxford, 1986.

[ReWe98] H.L. Resnikoff and R.O. Wells, Wavelet Analysis: The Scalable Structure of Information, Springer-Verlag, New York, 1998.

[Vai93] P.P. Vaidyanathan, Multirate Systems and Filter Banks, Prentice Hall, Englewood Cliffs, NJ, 1993. NORWAY

Department of Mathematics, University of Oslo, PB 1053 - Blindern, N-0316 Oslo,

E-mail address: bratteli@math.uio.no

Department of Mathematics, The University of Iowa, 14 Maclean Hall, Iowa City, IA 52242-1419, U.S.A.

E-mail address: jorgen@math.uiowa.edu 